Mon. Not. R. Astron. Soc. 000, ??-?? () Printed 30 July $2018 \quad$ (MN LATEX style file v2.2)

\title{
Infrared dust bubble CS51 and its interaction with the surrounding interstellar medium
}

\author{
Swagat R Das ${ }^{1 \star}$, Anandmayee Tej ${ }^{1}$, Sarita Vig ${ }^{1}$, Hong-Li Liu ${ }^{2,3,4}$, Tie Liu ${ }^{5}$ \\ Ishwara Chandra C.H. ${ }^{6}$, Swarna K. Ghosh ${ }^{6}$ \\ ${ }^{1}$ Indian Institute Of Space Science And Technology, Trivandrum, India \\ 2 Department of Physics, The Chinese University of Hong Kong, Shatin, NT, Hong Kong SAR \\ 3 Departamento de Astronomía, Universidad de Concepción, Av. Esteban Iturra s/n, Distrito Universitario, 160-C, Chile \\ 4 Chinese Academy of Sciences, South America Center for Astrophysics, Camino El Observatorio 1515, Las Condes, Santiago, Chile \\ ${ }^{5}$ Korea Astronomy and Space Science Institute 776, Daedeokdae-ro, Yuseong-gu, Daejeon, Republic of Korea 305-348 \\ ${ }^{6}$ National Centre for Radio Astrophysics (NCRA-TIFR), Pune, India
}

\begin{abstract}
A multiwavelength investigation of the southern infrared dust bubble CS51 is presented in this paper. We probe the associated ionized, cold dust, molecular and stellar components. Radio continuum emission mapped at 610 and $1300 \mathrm{MHz}$, using the Giant Metrewave Radio Telescope, India, reveal the presence of three compact emission components (A, B, and C) apart from large-scale diffuse emission within the bubble interior. Radio spectral index map show the coexistence of thermal and non-thermal emission components. Modified blackbody fits to the thermal dust emission using Herschel PACS and SPIRE data is performed to generate dust temperature and column density maps. We identify five dust clumps associated with CS51 with masses and radius in the range $810-4600 \mathrm{M}_{\odot}$ and $1.0-1.9 \mathrm{pc}$, respectively. We further construct the column density probability distribution functions of the surrounding cold dust which display the impact of ionization feedback from high-mass stars. The estimated dynamical and fragmentation timescales indicate the possibility of collect and collapse mechanism in play at the bubble border. Molecular line emission from the MALT90 survey is used to understand the nature of two clumps which show signatures of expansion of CS51.
\end{abstract}

Key words: stars: formation - ISM: HII region - ISM - radio continuum - ISM: individual objects (CS51-IRAS 17279-3350)

\section{INTRODUCTION}

High-mass stars $\left(\mathrm{M} \gtrsim 8 \mathrm{M}_{\odot}\right)$ are known to have significant influence on the surrounding interstellar medium (ISM) given their radiative, mechanical and chemical feedback. The last decade has seen tremendous progress towards understanding their formation and interaction with the ISM (see reviews by Zinnecker \& Yorke 2007, Tan et al. 2014). A combination of thermal pressure of the expanding HII region, powerful stellar wind, and radiation pressure associated with a newly formed massive star mostly results in a 'bubble'. This manifests as a shell of enhanced density of swept up gas and dust between the ionization and the shock fronts encompassing a relatively low-density, evacuated cavity around the central star. The 'bubbles' display bright-rimmed midinfrared (MIR) morphology (Weaver et al. 1977, Churchwell et al. 2006, 2007, Watson et al. 2008, Deharveng et al. 2010
Anderson et al. 2012 Kendrew et al.|2012 Ochsendorf et al. 2014). The MIR emission is attributed to polycyclic aromatic hydrocarbon $(\mathrm{PAH})$ molecules in the photodissociation regions (PDRs) surrounding $\mathrm{O}$ and early-B stars. Catalogs of infrared (IR) bubbles (Churchwell et al. 2006 2007; Simpson et al. 2012) show that these features are ubiquitous in our Galaxy. The last few years have seen a plethora of papers on the nature of these bubbles and also on the formation of a new generation of stars in their adjacent shells or bright rimmed clumps (Urquhart et al. 2007, Deharveng et al. 2010: Dewangan et al. 2012 | Liu et al. 2012 |Dewangan \& Ojha 2013| Hou \& Gao 2014: Ranjan Das et al. 2016| Baug et al. 2016 | Hattori et al. 2016 Liu et al. 2016 | 2017a b). An interesting statistical study by Thompson et al. (2012) shows that the surface density of young stellar objects (YSOs) peak towards the projected angular radius of the bubbles. From the association of massive YSOs with the sample of bubbles studied, these authors further conclude that $\sim 14-30 \%$ of

\footnotetext{
* E-mail: swagat.12@iist.ac.in
} 
massive stars in the Milky Way would have possibly formed via the triggered star formation mode.

In this work, we focus on the southern IR bubble, CS51, which is centered at $\alpha_{2000}=17^{\mathrm{h}} 31^{\mathrm{m}} 14.90^{\mathrm{s}}, \delta_{2000}=$ $-33^{\circ} 52^{\prime} 55.00^{\prime \prime}$ and displays a closed ring morphology with an effective radius of 2.1' (Churchwell et al. 2007. Simpson et al. 2012). A bright IRAS source, IRAS 17279-3350, with bolometric luminosity of $1.6 \times 10^{5} \mathrm{~L}_{\odot}$ (Beltrán et al. 2006) is found to be associated with the bubble. Several kinematic distance estimates to this source are found in the literature. The near and far distance estimates vary between $5.1-$ $6.5 \mathrm{kpc}$ and 11.7 - $13.4 \mathrm{kpc}$, respectively (Caswell \& Haynes 1987, Peeters et al. 2002, Beltrán et al. |2006 |Churchwell et al. 2007; Sánchez-Monge et al. 2013a: Urquhart et al. 2013). In this work, we have adopted the near kinematic distance of $5.3 \mathrm{kpc}$ from Churchwell et al. (2007). The justification for adopting the near kinematic distance is the presence of Hi self-absorption towards the $\mathrm{CO}$ emission peak as observed by Garwood \& Dickey (1989) (see Fig. 1 of their paper for source G354.188-0.073 which is associated with the bubble). This feature resolves the kinematic distance ambiguity and places objects at the near distance (Kolpak et al. 2003: Roman-Duval et al. 2009).

The region associated with the bubble has been probed in radio bands by a few authors. Radio continuum observations at 4.8 and $8.6 \mathrm{GHz}$ by Martín-Hernández, van der Hulst \& Tielens (2003) reveal the presence of three components (named A, B, and C), with A being associated with IRAS point source. These components are also studied at 5-GHz (Becker et al. 1994) and at $1.4 \mathrm{GHz}$ (Zoonematkermani et al. 1990). From the 1.2-mm observation, using the SEST 15-m telescope, Beltrán et al. (2006) identified two massive dust clumps of masses 665 and $764 \mathrm{M}_{\odot}$. The peak positions of these clumps correlate with that of the components $A$ and $\mathrm{C}$, respectively. Non-detection of $\mathrm{H}_{2} \mathrm{O}, \mathrm{OH}$ and $\mathrm{CH}_{3} \mathrm{OH}$ masers towards IRAS 17279-3350 was reported in Chan, Henning \& Schreyer (1996) and references therein. However, in a later survey, the $6.7-\mathrm{GHz}$ methanol maser was detected towards component C (Caswell et al. 2010).

Figure 1 shows the MIR and near-infrared (NIR) view of the region associated with CS51. The displayed morphology in the MIR is similar to those observed in other bubbles (Watson et al. 2008, Ji et al. 2012, Sidorin et al. 2014; Liu et al. 2016). 24- $\mu \mathrm{m}$ emission sampling the hot dust is enclosed within the bright-rimmed $8-\mu \mathrm{m}$ emission which shows a broken morphology towards the south. The $8-\mu \mathrm{m}$ emission is seen to be extended in the form of an envelope beyond the identified bubble boundary. However, the NIR colourcomposite image does not reveal any bubble signature and the region is seen to be densely populated. A faint, compact $\mathrm{K}_{\mathrm{s}}$-band nebulosity is seen towards IRAS 17279-3350 (see inset).

In presenting our study on the GLIMPSE bubble CS51, we have organized the paper in the following way. Section 2 deals with the radio continuum observation and associated data reduction procedure. In this section, we have also discussed the details of various archival data used in this study. In Section 3 , we discuss the results obtained using various datasets. Section 4 focuses on the feedback of the high-mass star responsible for the formation of CS51 and its impact on the surrounding ISM and in Section 5, we summarize our results.

\section{OBSERVATIONS AND ARCHIVAL DATA SETS}

\subsection{Radio Continuum Observations}

To probe the ionized gas associated with the bubble, we have carried out low-frequency radio continuum observations at 610 and $1300 \mathrm{MHz}$ with a bandwidth of $32 \mathrm{MHz}$ using Giant Metrewave Radio Telescope (GMRT), Pune India. GMRT has a hybrid configuration with 30 fully steerable antennae each of 45-m diameter placed in a ' $\mathrm{Y}$ ' shaped array (for details about GMRT, see Swarup et al. 1991). The central square of GMRT contains 12 antennae randomly distributed within $1 \times 1 \mathrm{~km}^{2}$, with the lowest baseline, $\sim 100 \mathrm{~m}$, sensitive to diffuse emission. The remaining 18 antennae are located on the arms with 6 on each arm. This renders the array capable of producing high angular resolution with the largest baseline of $\sim 25 \mathrm{~km}$.

The radio observations were carried out in the spectral line mode to minimize the effects of bandwidth smearing and narrow-band RFI. Radio sources 3C48 and 3C286 were used as primary flux calibrators and 1714-252 was used as phase calibrator for estimating the amplitude and phase gains for flux and phase calibration of the measured visibilities. The continuum data reduction is carried out using the Astronomical Image Processing Software (AIPS). The data sets are carefully scrutinized to identify bad data (dead antenna, bad baselines, RFI, spikes, etc.) using the tasks UVPLT and TVFLG. Subsequent editing of these are carried out using the tasks TVFLG and UVFLG. To keep the bandwidth smearing effect negligible the data sets are averaged in frequency using the task SPLAT. Low-frequency radio maps are generated by Fourier inversion and subsequent cleaning using the task IMAGR, in which we set the 'robustness' parameter to +1 (on a scale, where +4 represents nearly natural weighting and -4 is close to uniform weighting of the baselines). Wide-field imaging technique is used in order to account for the $w$-term effect (non-coplanarity). Several iterations of 'phase-only' and a final iteration of 'amplitude and phase' self calibration are performed to minimize the amplitude and phase errors and to reduce the rms noise in the maps. The final maps are then primary beam corrected using the task PBCOR.

Galactic diffuse emission contributes to the rise in system temperature, while observing close to the Galactic plane. At the low frequencies of our radio observations (especially at $610-\mathrm{MHz}$ ), the contribution from the Galactic diffuse emission becomes significant. Since the flux calibration is done using sources off the Galactic plane, appropriate scaling of flux densities in the final images is essential. The scaling factor is estimated under the assumption that the Galactic diffuse emission follows a power-law spectrum and $T_{s k y}$ at frequency $\nu$ for the target position is determined using the following equation

$$
T_{s k y}=T_{s k y}^{408}\left(\frac{\nu}{408 \mathrm{MHz}}\right)^{\gamma}
$$

where, $\gamma$ is the spectral index of the Galactic diffuse emission and is taken as -2.55 (Roger et al. 1999). Here, the sky temperature, $T_{s k y}$, is estimated by using the measurements obtained from the all-sky 408-MHz survey of Haslam et al. (1982). This yields scaling factors of 2.26 and 1.27 for 

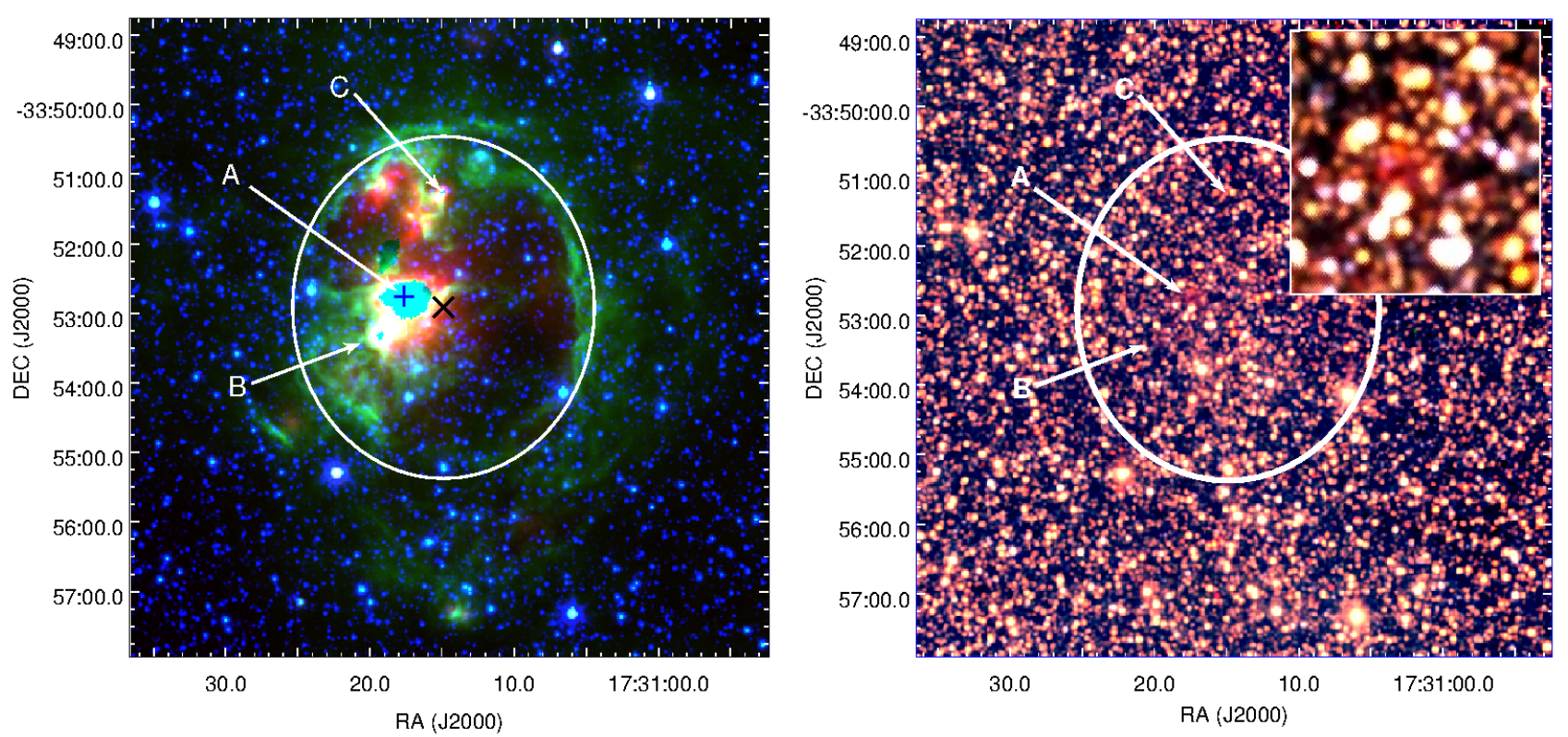

Figure 1. IR colour-composite images of the region towards CS51. Left: The IRAC-MIPSGAL MIR colour-composite image with $24 \mu \mathrm{m}$ (red), $8 \mu \mathrm{m}$ (green) and $4.5 \mu \mathrm{m}$ (blue). The cross mark $(\times)$ shows the position of the bubble center (Churchwell et al. 2007. Simpson et al. 2012). The position of IRAS 17279-3350 associated with the bubble is shown as a ' + ' symbol in blue. The 24- $\mu \mathrm{m}$ emission is saturated at this location. The locations of the three identified components are also highlighted with arrows where the arrow heads are the positions of the components (Martín-Hernández, van der Hulst \& Tielens 2003). The ellipse shows the extent of the bubble as described Churchwell et al. (2007). Right: 2MASS NIR colour-composite image with $\mathrm{K}_{\mathrm{S}}$ band (red), $\mathrm{H}$ band (green) and J band (blue). The inset shows the faint nebulosity seen towards A.

Table 1. Details of the radio interferometric continuum observations towards CS51.

\begin{tabular}{lcc}
\hline \hline Details & $610 \mathrm{MHz}$ & $1300 \mathrm{MHz}$ \\
\hline Date of Obs. & 08 August 2014 & 14 August 2014 \\
Flux Calibrators & 3C286,3C48 & 3C286,3C48 \\
Phase Calibrators & $1714-252$ & $1714-252$ \\
Integration time & $\sim 5 \mathrm{hr}$ & $\sim 5 \mathrm{hr}$ \\
Synth. beam & $11.5^{\prime \prime} \times 9.9^{\prime \prime}$ & $9.9^{\prime \prime} \times 6.5^{\prime \prime}$ \\
Position angle. (deg) & 29.8 & 64.7 \\
$r m s$ noise $($ mJy/beam) & 0.5 & 0.3 \\
\hline
\end{tabular}

610 and $1300 \mathrm{MHz}$, respectively. Details of the GMRT radio continuum observations and maps are given in Table 1

\subsection{Archival data sets}

\subsubsection{Near-infrared data from 2MASS}

NIR $\left(\mathrm{JHK}_{\mathrm{s}}\right)$ photometric data for point sources associated with CS51 are obtained from the Two Micron All Sky Survey (2MASS) ${ }^{1}$ (Skrutskie et al. 2006) Point Source Catalog. The 2MASS images have resolution of $\sim 5.0^{\prime \prime}$. Source selection is based on the "read-flag" that gives the uncertainty in the

\footnotetext{
1 This publication makes use of data products from the Two Micron All Sky Survey, which is a joint project of the University of Massachusetts and the Infrared Processing and Analysis Center/California Institute of Technology, funded by the NASA and the NSF.
}

magnitude. In our sample, we retain only those sources with "read-flag" value of 2. The 2MASS data are used to study the nature of the stellar population which are likely to be associated with the bubble.

\subsubsection{Mid-infrared data from Spitzer}

MIR data of the region towards CS51 have been obtained from the archives of the Spitzer Space Telescope. Photometric data and images at wavelengths of 3.6, 4.5, 5.8, $8.0 \mu \mathrm{m}$ have been retrieved from the Galactic Legacy Infrared Midplane Survey Extraordinaire (GLIMPSE; Benjamin et al. 2003). These images have resolution of $<2^{\prime \prime}$. We have obtained photometric data in IRAC bands from the 'highly reliable' catalog of GLIMPSE II Spring'08 catalog. The 24$\mu \mathrm{m}$ image is obtained from the MIPSGAL survey (Rieke et al. 2004) and has a resolution of $\sim 6^{\prime \prime} .24-\mu \mathrm{m}$ photometric data is extracted from the MIPSGAL catalog (Gutermuth \& Heyer 2015). Using these data, the structure of the bubble and distribution of YSOs are investigated.

\subsubsection{Far-infrared data from Herschel}

Far-infrared (FIR) data for the bubble are obtained from the Herschel Space Observatory archives. The images obtained were observed as part of the Herschel infrared Galactic plane Survey (HI-GAL; Molinari et al. 2010). We have used the images at wavelengths $70-500 \mu \mathrm{m}$ obtained from the Photodetector Array Camera and Spectrometer (PACS; Poglitsch et al. 2010) and Spectral and Photometric Imaging Receiver (SPIRE; Griffin et al. 2010). The images have resolutions of $5,11.4,17.9,25$ and $35.7^{\prime \prime}$ at $70,160,250,350$, and 
$500 \mu \mathrm{m}$, respectively. We have used the FIR data to study the physical properties of cold dust emission associated with the bubble.

\subsubsection{Molecular line data from MALT90 survey}

MALT90 (Millimeter Astronomy Legacy Team 90 GHz) survey was aimed in part at characterizing the molecular clumps in the vicinity of HII regions. The survey was carried out using the ATNF Mopra 22-m telescope with simultaneous mapping of 16 molecular lines near $90 \mathrm{GHz}$ with a spectral resolution of $0.11 \mathrm{~km} \mathrm{~s}^{-1}$. The source list was taken from the ATLASGAI $270-\mu \mathrm{m}$ continuum survey which detects both cold and warm clumps. We have retrieved the data from the MALT90 site (http://malt90.bu.edu). The details regarding the survey can be found in Foster et al. (2011), Foster et al. (2013) and Jackson et al. (2013). Data reduction was performed using GILDAS (Grenoble Image and Line Data Analysis Software $)^{3}$ We mainly explore the physical properties of the cold dust clumps using this data.

\section{RESULTS AND DISCUSSION}

\subsection{Ionized emission}

Radio emission, mapped at 610 and $1300 \mathrm{MHz}$, probing the ionized gas associated with CS51 is shown in Figure 2 Emission at both frequencies display complex morphology. Apart from an elongated cavity (a void in emission) seen towards the south-west, the bubble interior is filled with diffuse emission. The cavity is much more pronounced in the $1300 \mathrm{MHz}$ map. This gives the radio emission a broken shell-like morphology. Faint, diffuse emission extends beyond the identified bubble rim especially towards the south. The maps also reveal three compact regions of enhanced emission. Out of these, one is closer to the likely centre of the bubble, the second (and brightest of them) is more towards the southeast periphery and the third one is located in the north-west rim of the bubble. The peak positions of these components at 610 and $1300 \mathrm{MHz}$ coincide within $\sim 4^{\prime \prime}$.

The overall structure described above is consistent with the 4.8 and $8.6-\mathrm{GHz}$ maps obtained using the Australian Telescope Compact Array (ATCA) and presented in MartínHernández, van der Hulst \& Tielens (2003). Their 4.8-GHz map shows patchy, diffuse emission extending in the northsouth direction. Spatial correlation with the MIR images clearly shows that most of this emission lies within the confines of the bubble. As mentioned in the introduction, Martín-Hernández, van der Hulst \& Tielens (2003) have also identified three distinct components namely $\mathrm{A}, \mathrm{B}$, and $\mathrm{C}$ with $\mathrm{A}$ being extended and $\mathrm{B}$ and $\mathrm{C}$ as compact. The components $\mathrm{B}$ and $\mathrm{C}$ appear detached from the main complex A. The locations of these components agree excellently (within $2.5^{\prime \prime}$ from the peak positions in the $1300-\mathrm{MHz}$ map)

2 ATLASGAL is a collaboration between the Max Planck Gesellschaft (MPG: Max Planck Institute for Radioastronomy, Bonn and the Max Planck Institute for Astronomy, Heidelberg), the European Southern Observatory (ESO) and the University of Chile.

3 https://www.iram.fr/IRAMFR/GILDAS/ with the peaks of the detected compact components in the GMRT maps and hence we retain the same nomenclature. Their higher resolution 8.6-GHz map probes only the core of component $\mathrm{A}$ as most of the diffuse emission is resolved out. The flux densities and the sizes of the bubble as well as the three components determined from the GMRT and ATCA maps are listed in Table 2 To estimate the flux and size of the components, we use the following procedure outlined in Nandakumar et al. (2016). We first determine the threshold above which the diffuse emission becomes significant. Starting from the peak flux density, we sequentially plot contours as a percentage of the peak flux density and go upto the level where the contribution from the diffuse emission becomes apparent. This occurs at 40, 20, and $50 \%$ of the peak flux density for the components A, B, and C, respectively. Next, this threshold emission level is given as input in the 2D clumpfind code of Williams, de Geus \& Blitz (1994) to determine the sizes and integrated flux densities of the components which are considered as individual clumps in this algorithm. The size is taken as the geometric mean of the full width half maximum $(F W H M)$ for x-axis, $F W H M_{x}$ and for y-axis, $F W H M_{y}$. It should be noted here that completely decoupling the contribution of diffuse emission is difficult. Except for a larger (factor of $\sim 3$ ) peak flux density for component A, our estimated values of peak and integrated flux densities of the three components at $1300 \mathrm{MHz}$ are consistent with that obtained by Zoonematkermani et al. (1990) at $1400 \mathrm{MHz}$.

We derive various physical parameters associated with the ionized emission of the bubble. Assuming the radio emission at $1300 \mathrm{MHz}$ to be optically thin and emanating from a homogeneous, isothermal medium, we derive the Lyman continuum flux $\left(\mathrm{N}_{\mathrm{lyc}}\right)$ required to maintain ionization in the nebula, the number density of electrons $\left(n_{e}\right)$ and the emission measure (EM) using the following expressions (Schmiedeke et al. 2016)

$$
\begin{gathered}
\left(\frac{\mathrm{N}_{\mathrm{Lyc}}}{\mathrm{sec}^{-1}}\right)=4.771 \times 10^{42}\left(\frac{\mathrm{S}_{\nu}}{\mathrm{Jy}}\right)\left(\frac{\mathrm{T}_{\mathrm{e}}}{\mathrm{K}}\right)^{-0.45} \\
\times\left(\frac{\nu}{\mathrm{GHz}}\right)^{0.1}\left(\frac{\mathrm{D}}{\mathrm{pc}}\right)^{2} \\
\left(\frac{\mathrm{n}_{\mathrm{e}}}{\mathrm{cm}^{-3}}\right)=2.576 \times 10^{6}\left(\frac{\mathrm{S}_{\nu}}{\mathrm{Jy}}\right)^{0.5}\left(\frac{\mathrm{T}_{\mathrm{e}}}{\mathrm{K}}\right)^{0.175} \\
\times\left(\frac{\nu}{\mathrm{GHz}}\right)^{0.05}\left(\frac{\theta_{\text {source }}}{\operatorname{arcsec}}\right)^{-1.5}\left(\frac{\mathrm{D}}{\mathrm{pc}}\right)^{-0.5} \\
\left(\frac{\mathrm{EM}}{\mathrm{pc} \mathrm{cm}-6}\right)=3.217 \times 10^{7}\left(\frac{\mathrm{S}_{\nu}}{\mathrm{Jy}}\right)\left(\frac{\mathrm{T}_{\mathrm{e}}}{\mathrm{K}}\right)^{0.35} \\
\times\left(\frac{\nu}{\mathrm{GHz}}\right)^{0.1}\left(\frac{\theta_{\text {source }}}{\text { arcsec }}\right)^{-2}
\end{gathered}
$$

where, $\mathrm{N}_{\text {lyc }}$ is the Lyman continuum photons per second, $\mathrm{T}_{\mathrm{e}}$ is the electron temperature, $\nu$ is the frequency, $\mathrm{S}_{\nu}$ is the integrated flux, $\mathrm{D}$ is the kinematic distance to the bubble, $\theta$ refers to the angular size of the region considered (the entire bubble or the components). We determine the electron temperature adopting the following expression (Quireza et al. 2006

$$
\mathrm{T}_{\mathrm{e}}=(5780 \pm 350)+(287 \pm 46) \mathrm{R}_{\mathrm{G}}
$$



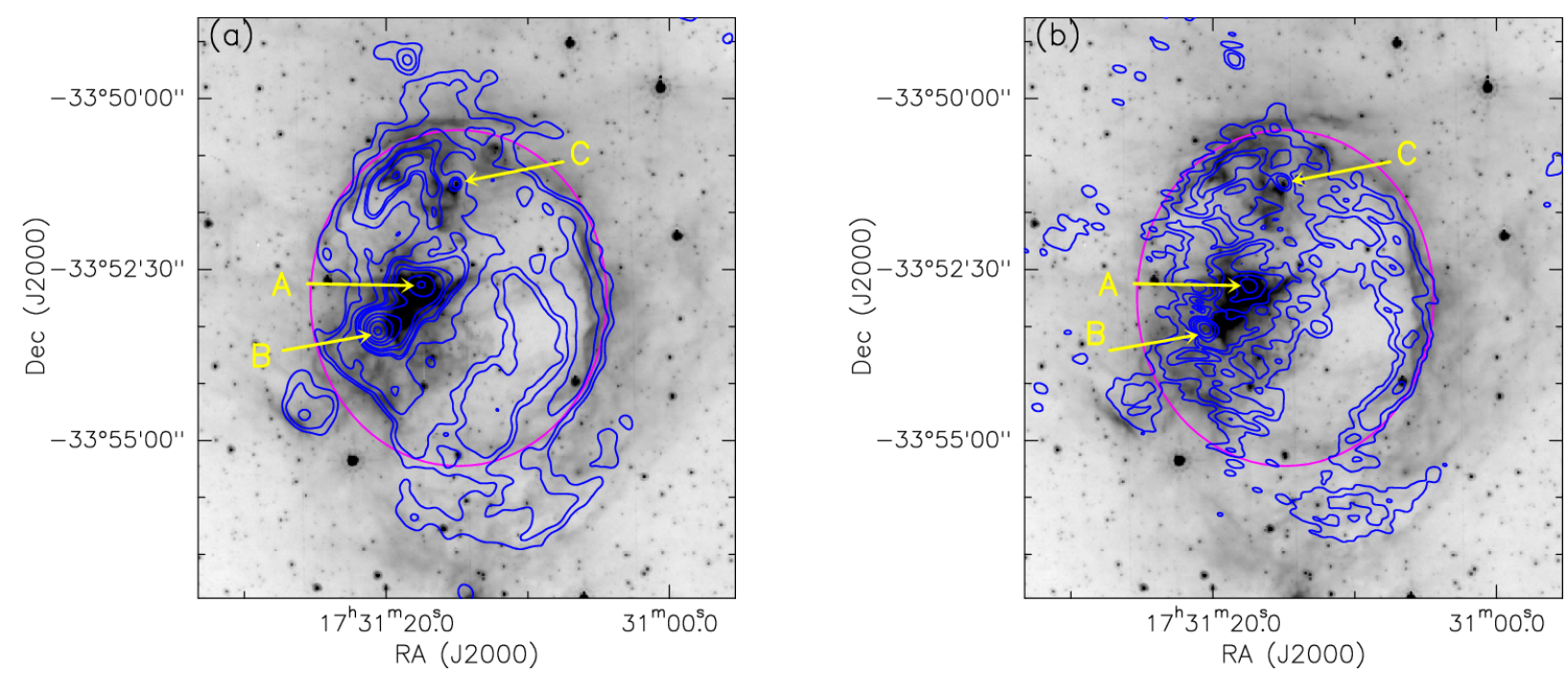

Figure 2. Ionized emission associated with the bubble CS51. Left: Radio continuum emission at $610 \mathrm{MHz}$ associated with CS51 shown as contours overlaid on the 8- $\mu \mathrm{m}$ IRAC image. Right: Same as the left figure for the $1300-\mathrm{MHz}$ emission. In both the figures, the contour levels are $0.3,1.0,2.5,5,12,25,40,60$, and $90 \%$ of the peak values. The peak fluxes at 610 and $1300 \mathrm{MHz}$ are 0.34 and $0.38 \mathrm{Jy} / \mathrm{beam}$, respectively. The positions of the identified compact components $\mathrm{A}, \mathrm{B}$, and $\mathrm{C}$ (see text) are marked. The magenta ellipse shows the extent of the bubble as described in Churchwell et al. (2007).

Table 2. GMRT and ATCA (Martín-Hernández, van der Hulst \& Tielens 2003) results. The peak coordinates, peak and integrated flux densities, and sizes of CS51 and the individual components are listed.

\begin{tabular}{|c|c|c|c|c|c|c|c|c|c|c|c|}
\hline & \multicolumn{2}{|c|}{ Peak Coordinates } & \multirow{2}{*}{$\frac{\text { Radius }}{(\operatorname{arcsec})}$} & \multicolumn{4}{|c|}{ Peak flux (Jy/beam) } & \multicolumn{4}{|c|}{ Integrated flux (Jy) } \\
\hline & $\begin{array}{c}\mathrm{RA}\left(\begin{array}{l}\mathrm{J} 2000) \\
\left(\begin{array}{ccc}h & m & s\end{array}\right)\end{array}\right.\end{array}$ & $\begin{array}{c}\operatorname{DEC}(\mathrm{J} 2000) \\
\left(\begin{array}{cc}0 & \prime \prime\end{array}\right)\end{array}$ & & $610 \mathrm{MHz}$ & $1300 \mathrm{MHz}$ & $4.8 \mathrm{GHz}$ & $8.6 \mathrm{GHz}$ & $610 \mathrm{MHz}^{\ddagger}$ & $1300 \mathrm{MHz}^{\ddagger}$ & $4.8 \mathrm{GHz}$ & $8.6 \mathrm{GHz}$ \\
\hline $\begin{array}{c}\text { CS51 } \\
\text { (entire bubble) }\end{array}$ & 173120.58 & -335324.39 & $126^{\dagger}$ & 0.34 & 0.38 & - & - & $5.62 \pm 0.56$ & $4.07 \pm 0.40$ & - & - \\
\hline \multicolumn{12}{|c|}{ Components } \\
\hline A & $1731 \quad 17.53$ & $\begin{array}{lll}-33 & 52 & 43.39\end{array}$ & $7.3^{*}$ & 0.15 & 0.13 & 0.02 & 0.01 & $0.35 \pm 0.03$ & $0.45 \pm 0.04$ & - & 0.52 \\
\hline B & 173120.58 & -335324.39 & $4.8^{*}$ & 0.34 & 0.38 & 0.09 & 0.05 & $0.96 \pm 0.09$ & $0.51 \pm 0.05$ & 0.10 & 0.06 \\
\hline $\mathrm{C}$ & $1731 \quad 15.04$ & $-3351 \quad 14.40$ & $4.1^{*}$ & 0.02 & 0.03 & 0.08 & 0.05 & $0.03 \pm 0.003$ & $0.04 \pm 0.004$ & 0.12 & 0.11 \\
\hline
\end{tabular}

$\dagger$ Effective radius from Churchwell et al. 2007, * Estimated from $2 \mathrm{D}$ clumpfind algorithm output, $\ddagger$ Error in integrated flux has been calculated following the equation from Sánchez-Monge et al. (2013b] $\left[\left(2 \sigma\left(\theta_{\text {source }} / \theta_{\text {beam }}\right)^{1 / 2}\right)^{2}+\left(2 \sigma_{\text {flux }} \text {-scale }\right)^{2}\right]^{1 / 2}$, where $\sigma$ is the $r m s$ noise level of the map, $\theta_{\text {source }}$ and $\theta_{\text {beam }}$ are the size of the source and the beam, respectively, and $\sigma_{\text {flux }}$ scale is the error in the flux scale, which takes into account the uncertainty on the calibration applied to the integrated flux of the source. For GMRT maps, uncertainty in the flux calibration is taken to be $5 \%$ Lal \& Rao 2007).

From the estimated Galactocentric distance $\left(R_{G}\right)$ of $3.3 \mathrm{kpc}$ to CS51, we determine the electron temperature to be $6700 \pm 380 \mathrm{~K}$. This is higher than the value of $5300 \mathrm{~K}$ quoted in Caswell \& Haynes (1987) derived using radio recombination line measurements. These authors derived the electron temperature for the radio source, which is located at an angular distance of $\sim 43^{\prime \prime}$ from the IRAS source. For further analysis, we use the electron temperature estimate of $6700 \pm 380 \mathrm{~K}$. Table 3 lists the derived radio properties of the bubble as well as the three components. The table also lists results from Martín-Hernández, van der Hulst \& Tielens (2003).

The estimated Lyman continuum photon flux of $1.1 \times$ $10^{49} \mathrm{~s}^{-1}$ indicates that atleast one $\mathrm{O} 6 \mathrm{~V}-\mathrm{O} 5.5 \mathrm{~V}$ star or a cluster of less massive stars are required to produce this flux (using Table 1 of Martins, Schaerer \& Hillier 2005) and maintain ionization in the nebula associated with the bubble CS51. This estimate is with the assumption of optically thin emission and hence serves as a lower limit as the emission could be optically thick at $1300 \mathrm{MHz}$. Various studies in the literature have also shown that dust absorp- tion of Lyman continuum photons can be very high Inoue 2001. Arthur et al. 2004, Paron, Petriella \& Ortega 2011). Assuming a uniform distribution and taking the derived value of electron density as a representative average value for the entire bubble, we estimate the mass of ionized gas $\left(\mathrm{M}_{\mathrm{ion}}=\frac{4}{3} \pi \mathrm{r}_{\mathrm{Hii}}^{3} \mathrm{n}_{\mathrm{e}} \mathrm{m}_{\mathrm{p}}\right.$, where $\mathrm{r}_{\mathrm{Hii}}$ is taken to be the radius of the bubble and $\mathrm{m}_{\mathrm{p}}$ is mass of proton) to be $\sim 300 \mathrm{M}_{\odot}$. However, it should be noted that some ionized gas is seen beyond the defined bubble radius and its contribution has not been taken into account.

The components $\mathrm{A}, \mathrm{B}$, and $\mathrm{C}$ could be harbouring massive stars and thus internally ionized. The derived Lyman continuum photon flux values suggest spectral types of O9 O8.5, O8.5 - O8, and B0.5 - B0 for A, B, and C, respectively. Component $\mathrm{C}$ is more likely internally heated as there exists a $70 \mu \mathrm{m}$ point source (Marton et al. 2017) coinciding with the peak position. However, for components A and B we cannot rule out the possibility of these being externally ionized clumps due to density inhomogeneities. Such compact ionized objects are seen in several extended HiI regions (Garay et al. 1998, Kim \& Koo 2003, 2001). 
Table 3. Physical parameters of the bubble and the three components. The values given in parenthesis are from Martín-Hernández, van der Hulst \& Tielens (2003).

\begin{tabular}{|c|c|c|c|c|}
\hline & $\begin{array}{l}\log \mathrm{N}_{\mathrm{lyc}} \\
\left(\mathrm{sec}^{-1}\right)\end{array}$ & $\begin{array}{c}\mathrm{n}_{\mathrm{e}} \\
\left(\times 10^{3} \mathrm{~cm}^{-3}\right)\end{array}$ & $\begin{array}{c}\mathrm{EM} \\
\left(\times 10^{6} \mathrm{pc} \mathrm{cm}^{-6}\right)\end{array}$ & Spectral Type \\
\hline $\begin{array}{c}\text { CS51 } \\
\text { (entire bubble) }\end{array}$ & 49.03 & 0.08 & 0.04 & $\mathrm{O} 6 \mathrm{~V}-\mathrm{O} 5.5 \mathrm{~V}$ \\
\hline \multicolumn{5}{|c|}{ Components } \\
\hline $\mathrm{A}$ & $48.07(47.88)$ & $2.00(1.10)$ & $1.50(0.6)$ & O9 - O8.5 \\
\hline $\mathrm{B}$ & $48.12(47.15)$ & $3.94(9.70)$ & $3.89(6.3)$ & O8.5- O8 \\
\hline $\mathrm{C}$ & $47.02(47.23)$ & $1.42(7.60)$ & $0.43(4.8)$ & $\mathrm{B} 0.5-\mathrm{B}^{a}$ \\
\hline
\end{tabular}

\subsection{Spectral index maps}

To probe the nature of the radio emission in the region associated with the bubble, we construct a spectral index map from the 610 and $1300 \mathrm{MHz}$ data. This requires a pixel-bypixel estimation of the spectral index, $\alpha$, defined as $\mathrm{S}_{\nu} \propto \nu^{\alpha}$, where $\mathrm{S}_{\nu}$ is the flux density at the frequency $\nu$. Since we are interested in studying the variation of the spectral index over the entire region associated with CS51, we need to sample the large-scale diffuse ionized emission. GMRT is not a scaled array between the observed frequencies implying that each frequency is sensitive to different spatial scales. Hence, to ensure that the contribution of diffuse emission is same at both frequencies, we generate a new map at $1300 \mathrm{MHz}$ from visibilities in the $u v$ range of $0.1-43 \mathrm{~K} \lambda$ which is consistent with the $u v$ coverage in the $610-\mathrm{MHz}$ map. The new $1300-\mathrm{MHz}$ map is regridded to the pixel size of $1^{\prime \prime}$ of the $610-\mathrm{MHz}$ map. Both maps are then convolved to a common lower resolution of $20^{\prime \prime} \times 20^{\prime \prime}$ to exclude any small scale statistical fluctuation in the estimated spectral index values. The above two steps are performed in the AIPS environment using the tasks LGEOM and CONVL. Retaining pixels above $5 \sigma$ ( $\sigma \sim 1 \mathrm{mJy} /$ beam $)$ in both maps, the spectral index map is obtained using the task COMB. The error map is also retrieved from the task output to get the pixel uncertainties. The spectral index map and the corresponding error map is shown in Figure 3 . The low-resolution $610-\mathrm{MHz}$ contours are overlaid on the spectral index map.

The spectral index values in the map vary between -1.75 to 1.5 and the estimated errors are less than $\sim 0.4$. However, a careful examination of the maps suggest that in the bubble interior the range is mostly between -1.0 to 1.0 with errors less than $\sim 0.1$. There are few patches showing extreme negative spectral index values and those are seen towards the eastern side of the component B and also towards the circular void seen north-west of component A. The observed range indicates the simultaneous presence of thermal freefree emission and non-thermal synchrotron emission which further suggest varying physical conditions. As discussed in Rodriguez et al. (1989), when only free-free emission and absorption mechanisms are involved, the spectral index lies between -0.1 and +2 . Spectral indices $<-0.25$ are attributed to non-thermal emission (Rosero et al. 2016 Curiel et al. 1993). Radio spectral index maps of several Galactic Hiı regions have shown similar co-existing thermal and nonthermal components (Russeil et al. 2016 Veena et al. 2016
Nandakumar et al. 2016). This has been attributed to shocks induced by outflows and/or winds. Such colliding wind interpretation is also cited for negative spectral indices seen towards Wolf-Rayet nebula (Benaglia et al. 2005).

In Table 4 , we list the spectral indices obtained from the map at the peak positions of A, B, and C. The table also gives the spectral index values obtained from the integrated flux densities. The later values for components $\mathrm{B}$ and $\mathrm{C}$ are fairly consistent with that obtained by Martín-Hernández, van der Hulst \& Tielens (2003). From the above estimates we infer thermal free-free emission to be associated with components $\mathrm{A}$ and $\mathrm{C}$ whereas the emission from component $\mathrm{B}$ shows spectral signature for non-thermal emission. Compact radio sources exhibiting such large negative spectral index have been discussed in Russeil et al. (2016) and Rodríguez et al. (2014) where the latter study puts forth a viable scenario of colliding winds of a massive binary system which produces a shocked region between the components where electrons can reach relativistic speeds by Fermi acceleration thus producing synchrotron emission. Similar interpretation has been discussed by Luque-Escamilla et al. (2011) for the extended non-thermal radio emission detected towards the W43 cluster. Protostars with an active magnetosphere could also give rise to such negative spectral indices (Deller, Forbrich \& Loinard 2013). Detailed study of component B is required to ascertain its exact nature.

\subsection{Identifying the ionizing stars}

A careful literature survey shows that there has been no identification of the ionizing source responsible for the HiI region associated with the bubble CS51. In this section we attempt to identify and propose the possible exciting source(s) responsible for CS51. As discussed in Churchwell et al. (2006, 2007), the association of HiI regions with IR bubbles indicate that these are formed by O- and/or B-type of stars. The Lyman continuum photon flux estimated from the GMRT radio maps suggest a spectral type of O6V - O5.5.

Identification of candidate exciting star(s) has been carried out in several bubbles based on MIR and NIR photometry of sources enclosed within the bubbles (Pomarès et al. 2009 |Petriella, Paron \& Giacani 2010, Ji et al. 2012 | Dewangan \& Ojha 2013 | Baug et al. 2016). We have followed the method adopted in a recent paper by Ortega et al. (2016). As we expect the candidate ionizing star(s) to be located within 

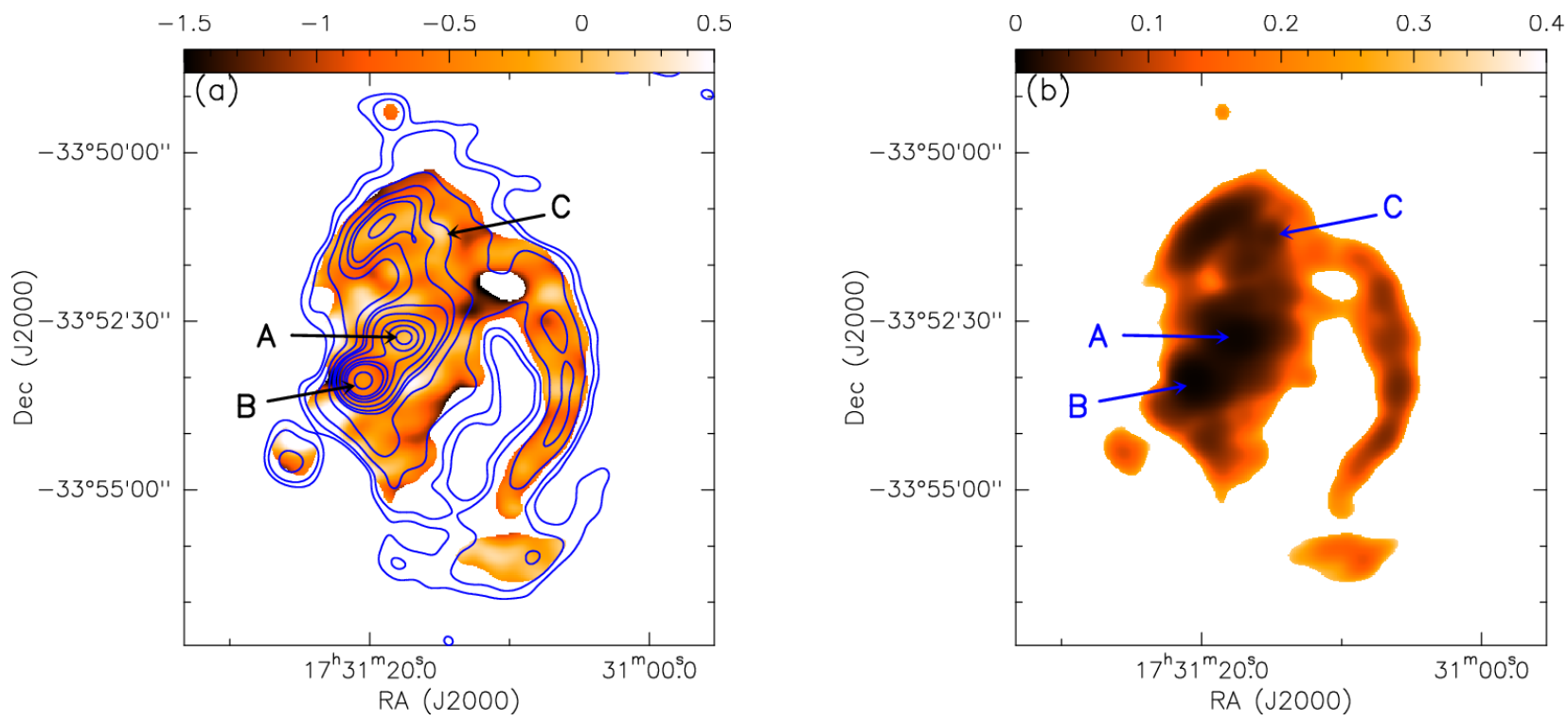

Figure 3. Left: Spectral index map of the region associated with CS51. The low-resolution $\left(20^{\prime \prime} \times 20^{\prime \prime}\right) 610-\mathrm{MHz}$ contours are overlaid. The contour levels are $0.5,1,2.5,5,10,15,25,35,45$ and $80 \%$ of the peak value $(0.67 \mathrm{mJy} /$ beam $)$. The components A, B, and C are marked. Right: The corresponding error map retrieved from the AIPS task COMB.

Table 4. Spectral indices of components A, B, and C.

\begin{tabular}{cccc}
\hline Components & \multicolumn{3}{c}{ Spectral Index } \\
\cline { 2 - 4 } & \multicolumn{2}{c}{ GMRT $610-1300 \mathrm{MHz}$} & ATCA $4.8-8.6 \mathrm{GHz}$ \\
\cline { 2 - 4 } & From map $^{a}$ & From Int. flux & From Int. flux \\
& & \\
\hline A & $-0.01 \pm 0.006$ & $0.19 \pm 0.06$ & - \\
B & $-0.33 \pm 0.004$ & $-0.78 \pm 0.06$ & -0.87 \\
C & $0.19 \pm 0.040$ & $-0.14 \pm 0.07$ & -0.16 \\
\hline
\end{tabular}

${ }^{a}$ relates to peak positions from spectral index map; ${ }^{b}$ obtained from the integrated flux densities of the final convolved $\left(20^{\prime \prime} \times 20^{\prime \prime}\right)$ maps; ${ }^{c}$ from Martín-Hernández, van der Hulst \& Tielens (2003).

the CS51 PDR, we consider 2MASS sources with good quality photometry (refer to Section 2.2.1) located within a radius of $2.1^{\prime}$ of the central coordinate of CS51. Inspite of the proper motion of the exciting star(s), they are still likely to be within the PDR given that these IR bubbles are young objects. However, they need not always be located at the geometrical centre of the bubble.

In our search for O stars within the CS51 PDR, we follow the BJHK colour criteria of Comerón \& Pasquali (2012). The criteria adopted by these authors is highly efficient in filtering out late-type contaminants. The B magnitudes of the retrieved sources are taken from the NOMAD1 catalog (Zacharias et al. 2004). Since the bubble CN20 studied by Ortega et al. (2016) is at a similar distance as CS51, we apply the same $\mathrm{K}_{\mathrm{s}}$ magnitude cut of 11 to exclude out reddened early-type stars in the background of CS51. This is based on the assumption of $A_{v} \sim 1$ per kpc of foreground extinction. We identify 17 sources satisfying the criteria of Comerón \& Pasquali (2012). These sources are marked in Figure 4 which shows the $8-\mu \mathrm{m}$ image overlaid with the contours showing the $610-\mathrm{MHz}$ radio emission. Except one, the sources fall in the region of the Class III population in the 2MASS colourcolour plot. The ones which have IRAC band magnitudes are also seen to populate the region occupied by Class III objects (Petriella, Paron \& Giacani 2010). In their study of similar IR bubbles, Ortega et al. (2016); Liu et al. (2016); Baug et al. (2016) have discussed the likely association of the location of the ionizing $\operatorname{star}(\mathrm{s})$ and bright $24-\mu \mathrm{m}$ emission in the bubble interior which also correlates with peak of the free-free radio emission. Following this argument, we identify five candidates labeled 1, 2, 3, 4, and 5 in Figure 4 Considering their associated visual extinctions from the $2 \operatorname{MASS}\left(\mathrm{H}-\mathrm{K}_{\mathrm{s}}\right)$ and $(\mathrm{J}-\mathrm{H})$ colour-colour plot, we derive their absolute $\mathrm{K}$ magnitudes and estimate the spectral type from Martins \& Plez (2006). The details of these five sources are given in Table 5. The location of star \#5 is towards the bubble periphery and hence it is less likely to qualify as a candidate exciting source. Similarly, star \#2 falls in the Class II YSO region in the 2MASS colour-colour plot. Thus, based on their nature and position inside the bubble, we identify sources \#1, 3 and 4 as the most promising candidates for ionization of CS51. Optical and NIR spectroscopy of these candidate exciting sources are required to qualify the above discussion. 
Table 5. Exciting star candidates associated with CS51.

\begin{tabular}{|c|c|c|c|c|c|c|c|c|c|c|c|}
\hline \# & $\begin{array}{c}\mathrm{RA} \\
\left(\begin{array}{ccc}h & m & s\end{array}\right)\end{array}$ & 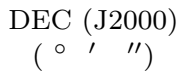 & $\begin{array}{c}\mathrm{B} \\
(\mathrm{mag})\end{array}$ & $\begin{array}{c}\mathrm{J} \\
(\mathrm{mag})\end{array}$ & $\begin{array}{c}\mathrm{H} \\
(\mathrm{mag})\end{array}$ & $\begin{array}{c}\mathrm{K} \\
(\mathrm{mag})\end{array}$ & $\mathrm{A}_{\mathrm{V}}$ & $\begin{array}{c}\mathrm{M}_{\mathrm{J}} \\
(\mathrm{mag})\end{array}$ & $\begin{array}{c}\mathrm{M}_{\mathrm{H}} \\
(\mathrm{mag})\end{array}$ & $\begin{array}{c}\mathrm{M}_{\mathrm{K}} \\
(\mathrm{mag})\end{array}$ & Spectral type \\
\hline 1 & 173116.01 & -335309.27 & 17.51 & 10.19 & 9.09 & 8.72 & 10.19 & -6.30 & -6.31 & -6.04 & $>\mathrm{O} 3 \mathrm{~V}$ \\
\hline 2 & 173117.56 & -335256.50 & 19.52 & 11.73 & 10.51 & 9.79 & 12.39 & -5.38 & -5.27 & -5.21 & $>\mathrm{O} 3 \mathrm{~V}$ \\
\hline 3 & 173117.88 & -335300.72 & 16.07 & 12.28 & 10.96 & 10.28 & 12.92 & -4.98 & -4.92 & -4.78 & $\mathrm{O} 4 \mathrm{~V}-\mathrm{O} 3 \mathrm{~V}$ \\
\hline 4 & 173118.58 & -335251.46 & 17.91 & 11.91 & 10.93 & 10.60 & 9.22 & -4.30 & -4.30 & -4.04 & $\mathrm{O} 6.5 \mathrm{~V}-\mathrm{O} 6 \mathrm{~V}$ \\
\hline 5 & 173120.17 & -335359.88 & 18.61 & 12.13 & 11.13 & 10.75 & 9.53 & -4.17 & -4.15 & -3.93 & $\mathrm{O} 7 \mathrm{~V}-\mathrm{O} 6.5 \mathrm{~V}$ \\
\hline
\end{tabular}

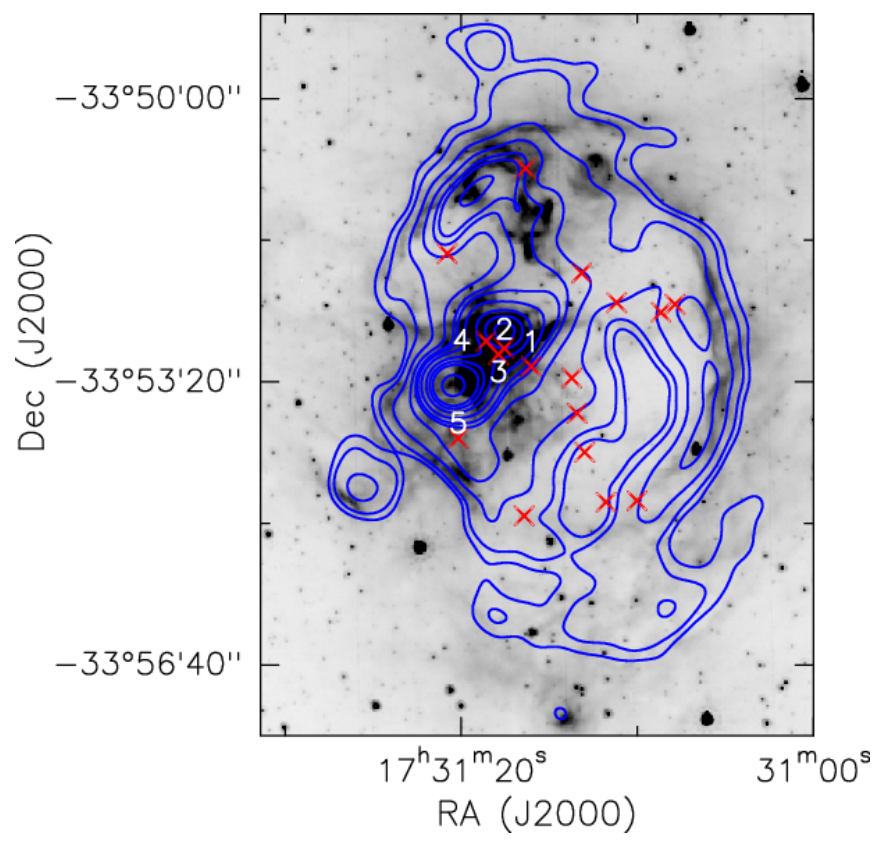

Figure 4. This figure displays the $8-\mu \mathrm{m}$ image on which is shown the distribution of O- and B-type stars (red crosses) in the bubble interior which satisfy the criteria discussed in Comerón \& Pasquali (2012). Low-resolution $610-\mathrm{MHz}$ contours are overlaid. The contour levels are same as in Figure 3 The candidate ionizing stars are labeled as \#1,2,3,4, and 5 . Refer to the text for further discussion.

\subsection{Emission from cold dust component}

We study the nature of the cold dust emission using the 160 $-500 \mu \mathrm{m}$ Herschel data. Line-of-sight average molecular hydrogen column density and dust temperature maps are generated by pixel-wise modified blackbody fits. In generating these maps we have excluded the $70-\mu \mathrm{m}$ emission following the discussion given in several studies (Peretto et al. 2010 Battersby et al. 2011, Peretto et al. 2010, Anderson et al. 2012). These studies reason that the emission at $70 \mu \mathrm{m}$ may not be optically thin and that there may be significant contribution from the warm dust components (e.g., very small dust grains, protostars) and hence cannot be modeled with a single temperature gray body. Thus we have only four points mostly on the Raleigh-Jeans part to constrain the model.

The initial steps involve converting the SPIRE map units from $\mathrm{MJy} \mathrm{sr}^{-1}$ to $\mathrm{Jy} \mathrm{pixel}^{-1}$ which is the unit of the PACS images. Subsequent to this, the 160, 250, 350- $\mu \mathrm{m}$ images are convolved and regridded to the lowest resolution $\left(35.7^{\prime \prime}\right)$ and largest pixel size $\left(14^{\prime \prime}\right)$ of the $500-\mu \mathrm{m}$ image.
The convolution kernels are taken from Aniano et al. (2011). The above steps are carried out using the Herschel data reduction software HIPE4

We estimate the background flux, $\mathrm{I}_{\mathrm{bg}}$, in each band from a relatively smooth and dark region devoid of bright, diffuse emission and filamentary structures. This region is located at an angular distance of $\sim 20^{\prime}$ from the bubble and centered at $\alpha_{2000}=17^{\mathrm{h}} 32^{\mathrm{m}} 14.46^{\mathrm{s}}, \delta_{2000}=-34^{\circ} 01^{\prime} 21.90^{\prime \prime}$. The background value was estimated by fitting a Gaussian function to the distribution of individual pixels in the specified region. The fitting was carried out iteratively by rejecting the pixels having values outside $\pm 2 \sigma$ till the fit converged (Battersby et al. 2011; Launhardt et al. 2013). We have used the same region for the determination of background offset in all the bands. $\mathrm{I}_{\mathrm{bg}}$ is estimated to be $-0.82,2.81,1.27$, and $0.45 \mathrm{Jy} \mathrm{pixel}^{-1}$ at $160,250,350$, and $500 \mu \mathrm{m}$, respectively. The negative flux value at $160 \mu \mathrm{m}$ is due to the arbitrary scaling of the PACS images.

We model the FIR emission with a modified blackbody that takes into consideration the optical depth, and the dust emissivity. As mentioned earlier, this is done pixelwise adopting the following functional form (Battersby et al. 2011. Faimali et al. 2012, Launhardt et al. 2013 | Mallick et al. 2015).

$$
\mathrm{S}_{\nu}(\nu)-\mathrm{I}_{\mathrm{bg}}(\nu)=\mathrm{B}_{\nu}\left(\nu, \mathrm{T}_{\mathrm{d}}\right) \Omega\left(1-\mathrm{e}^{-\tau_{\nu}}\right)
$$

and

$$
\tau_{\nu}=\mu_{\mathrm{H}_{2}} \mathrm{~m}_{\mathrm{H}} \kappa_{\nu} \mathrm{N}\left(\mathrm{H}_{2}\right)
$$

where, $\mathrm{S}_{\nu}(\nu)$ is the observed flux density, $\mathrm{I}_{\mathrm{bg}}(\nu)$ is the background flux, $\mathrm{B}_{\nu}\left(\nu, \mathrm{T}_{\mathrm{d}}\right)$ is the Planck's function, $\mathrm{T}_{\mathrm{d}}$ is the dust temperature, $\Omega$ is the solid angle (in steradians) from where the flux is obtained (solid angle subtended by a $14^{\prime \prime} \times 14^{\prime \prime}$ pixel), $\mu_{\mathrm{H}_{2}}$ is the mean molecular weight, $\mathrm{m}_{\mathrm{H}}$ is the mass of hydrogen atom, $\kappa_{\nu}$ is the dust opacity and $\mathrm{N}\left(\mathrm{H}_{2}\right)$ is the column density. We have assumed a value of 2.8 for $\mu_{\mathrm{H}_{2}}$ (Kauffmann et al. 2008). The dust opacity $\kappa_{\nu}$ is defined to be $\kappa_{\nu}=0.1(\nu / 1000 \mathrm{GHz})^{\beta} \mathrm{cm}^{2} / \mathrm{g}$, where, $\beta$ is the dust emissivity spectral index (Hildebrand 1983, Beckwith et al. 1990, André et al. 2010). The SED fitting was carried out using non-linear least square Levenberg-Marquardt algorithm, in which $\mathrm{T}_{\mathrm{d}}$ and $\mathrm{N}\left(\mathrm{H}_{2}\right)$ are kept as free parameters. Given the limited number of data points, we prefer to fix the value of $\beta$ to 2 (Hildebrand 1983. Beckwith et al.

4 The software package for Herschel Interactive Processing Environment (HIPE) is the application that allows users to work with the Herschel data, including finding the data products, interactive analysis, plotting of data, and data manipulation. 
1990, André et al. 2010 which is also a typical value used in studies related to IR dust bubbles (Anderson et al. 2012, Liu et al. 2016, Ortega et al. 2016). We have used a conservative $15 \%$ uncertainty on the background subtracted flux densities (Launhardt et al. 2013). The generated column density and temperature maps are shown in Figure 5, alongwith the corresponding $\chi^{2}$ map. We have overlaid the low resolution $\left(20^{\prime \prime} \times 20^{\prime \prime}\right) 610 \mathrm{MHz}$ radio map on the dust temperature map to correlate the ionized and dust components.

As seen in Figure 5 the pixel fits are good with $\chi^{2}<6$ in the map. The column density map shows a fragmented shell-like structure with low values tracing the inner region of the bubble. The bubble rim is seen to contain clumpy regions of enhanced column density. One clump is located relatively close to the centre of the bubble coincident with the radio peaks. Apart from this clump, the rest fit well into a picture of fragmented swept-up molecular shell. The peak column density $\left(3.6 \times 10^{22} \mathrm{~cm}^{-2}\right)$ is located towards the northern part of the bubble. Several clumpy structures and high density regions are also seen on the periphery and beyond the bubble. Correlating the ionized emission with the dust temperature map, we see high dust temperature towards the three components and the entire region associated with the bubble CS51 is clearly warmer than the surrounding region.

As shown in Figure 5, five cold dust clumps associated with CS51 are detected from the column density map. Instead of using the visual inspection followed by fitting ellipses to characterize the clumps (Liu et al. 2016), we use the astrodendro algorithm (Rosolowsky et al.|2008, Goodman et al. 2009). In doing so, we set a threshold column density of $8.0 \times 10^{21} \mathrm{~cm}^{-2}$ and detect clumps above this by specifying a minimum of 5 pixels for a potential clump.

Mass of the dust clumps is calculated using the following equation

$$
\mathrm{M}_{\text {clump }}=\mu_{\mathrm{H}_{2}} \mathrm{~m}_{\mathrm{H}} \mathrm{A}_{\text {pixel }} \Sigma \mathrm{N}\left(\mathrm{H}_{2}\right)
$$

where, $\mathrm{m}_{\mathrm{H}}$ is the mass of hydrogen, $\mathrm{A}_{\text {pixel }}$ is the pixel area in $\mathrm{cm}^{2}, \mu_{\mathrm{H}_{2}}$ is the mean molecular weight and $\Sigma \mathrm{N}\left(\mathrm{H}_{2}\right)$ is the integrated column density. The clump apertures retrieved from astrodendro are used to estimate the physical sizes $(\mathrm{r}=$ $(\mathrm{A} / \pi)^{0.5}$; Kauffmann \& Pillai 2010) $)$. The derived physical properties of the dust clumps are listed in Table 6] The volume number density is derived as, $\mathrm{n}_{\mathrm{H}_{2}}=3 \Sigma \mathrm{NH}_{2} / 4 \mathrm{r}$, $\mathrm{r}$ being the radius of the clump. The two clumps detected from the 1.2-mm emission by Beltrán et al. (2006) are likely to be associated with our Clump 3 and Clump 4. However, the masses derived in this paper are larger by a factor of 2 - 7 compared to the estimates of Beltrán et al. (2006). The possible reasons for this could be the different $\kappa_{\nu}$ and dust temperature values used by these authors and/or the large sizes of the clumps retrieved from the column density map. One also cannot exclude the effect of flux loss associated with ground based observations (Liu et al. 2017a).

\subsection{Associated stellar population}

To understand the star formation activity associated with CS51, we study the nature of the stellar population. We use Spitzer GLIMPSE and MIPSGAL data to identify and classify potential YSOs towards the bubble. For this we choose a region of radius $5^{\prime}$ centered on the bubble. We retrieve 1060 sources with photometric data in all the IRAC bands. We also explore the catalog of Gutermuth \& Heyer (2015) to include $24 \mu \mathrm{m}$ point sources which have $3.6 \mu \mathrm{m}$ photometry. From the distribution of IRAC sources extracted from the catalog, we see a dearth of point sources towards regions of bright MIR emission located in the bubble centre and periphery. Hence, to ensure a more complete sample of sources associated with the bubble we also make use of good photometric data from the 2MASS Point Source Catalog. A total of 1418 sources have good quality data in all three $\mathrm{JHK}_{\mathrm{s}}$ bands. The identification and classification of YSOs are carried out using the following procedures.

(a) This scheme uses the IRAC colors for the classification of YSOs as discussed in Allen et al. (2004). Class I (sources dominated by protostellar envelope emission) and Class II (sources dominated by protoplanetary disk) are segregated based on their location on the [3.6] - [4.5] vs [5.8] - [8.0] colour-colour plot (CCP), shown in Figure 6(a). The boxes on the plot demarcating the location of Class I and Class II sources, are adopted from Vig et al. (2007). Following this classification scheme, we have identified 21 YSOs, out of which 5 are Class I, 12 are Class II and 4 are Class I/II.

(b) In this method, we used the [3.6] - [24] vs [3.6] colourmagnitude plot (CMP) and the criteria discussed in Guieu et al. (2010) and Rebull et al. (2011) for the classification of YSOs. Out of 105 detected $24 \mu \mathrm{m}$ point sources, 63 have $3.6 \mu \mathrm{m}$ counterparts in the GLIMPSE highly reliable catalog. Figure 6(b) shows the colour-magnitude plot (CMP), where the vertical lines separate the YSOs of different evolutionary classes. The dotted curve on the plot marks the boundary of contaminated sources such as galaxies and diskless stars. Following this method, 20 YSOs are identified, out of which 9 are Class I, 8 are Class II, and 3 are flat-spectrum YSOs.

(c) In this procedure, YSOs are classified based on their IRAC spectral index. The IRAC spectral index $(\alpha=$ $d \log \left(\lambda F_{\lambda}\right) / d \log (\lambda)$; Lada 1987) is estimated for each source by a linear regression fit. Then the YSOs are classified into Class I $(\alpha>0)$ and Class II $(-2 \leqslant \alpha \leqslant 0)$, following the classification scheme of Chavarría et al. (2008). The distribution of YSOs following this classification scheme is shown in Figure 6(c). Here, we identify 38 YSOs out of which 8 are Class I and 30 are Class II sources.

(d) $\mathrm{JHK}_{\mathrm{s}}$ CCPs have been efficiently used by various authors (Sugitani et al. 2002, Ojha et al. 2004a b. Tej et al. 2006 Ji et al. 2012; Mallick et al.|2015) to identify YSOs. The CCP for the NIR sources associated with bubble CS51 is shown in Figure 6(d). The loci of main sequence (thin line) and giants (thick line) are taken from Bessell \& Brett (1988). The classical T Tauri locus shown as a long-dashed line is taken from Meyer, Calvet \& Hillenbrand (1997). The parallel dotted lines are the reddening vectors, on which the cross marks are the visual extinction placed at intervals of 5 mag. The short-dashed line represents the locus of Herbig AeBe sources is taken from Lada \& Adams (1992). We have assumed the interstellar reddening law from Rieke \& Lebofsky (1985). In this plot all the colours and curves are converted into Bessell \& Brett (1988) system. For better classification this plot is divided into three regions. The sources in ' $F$ ' region are field stars or Class III or Class II stars with small 


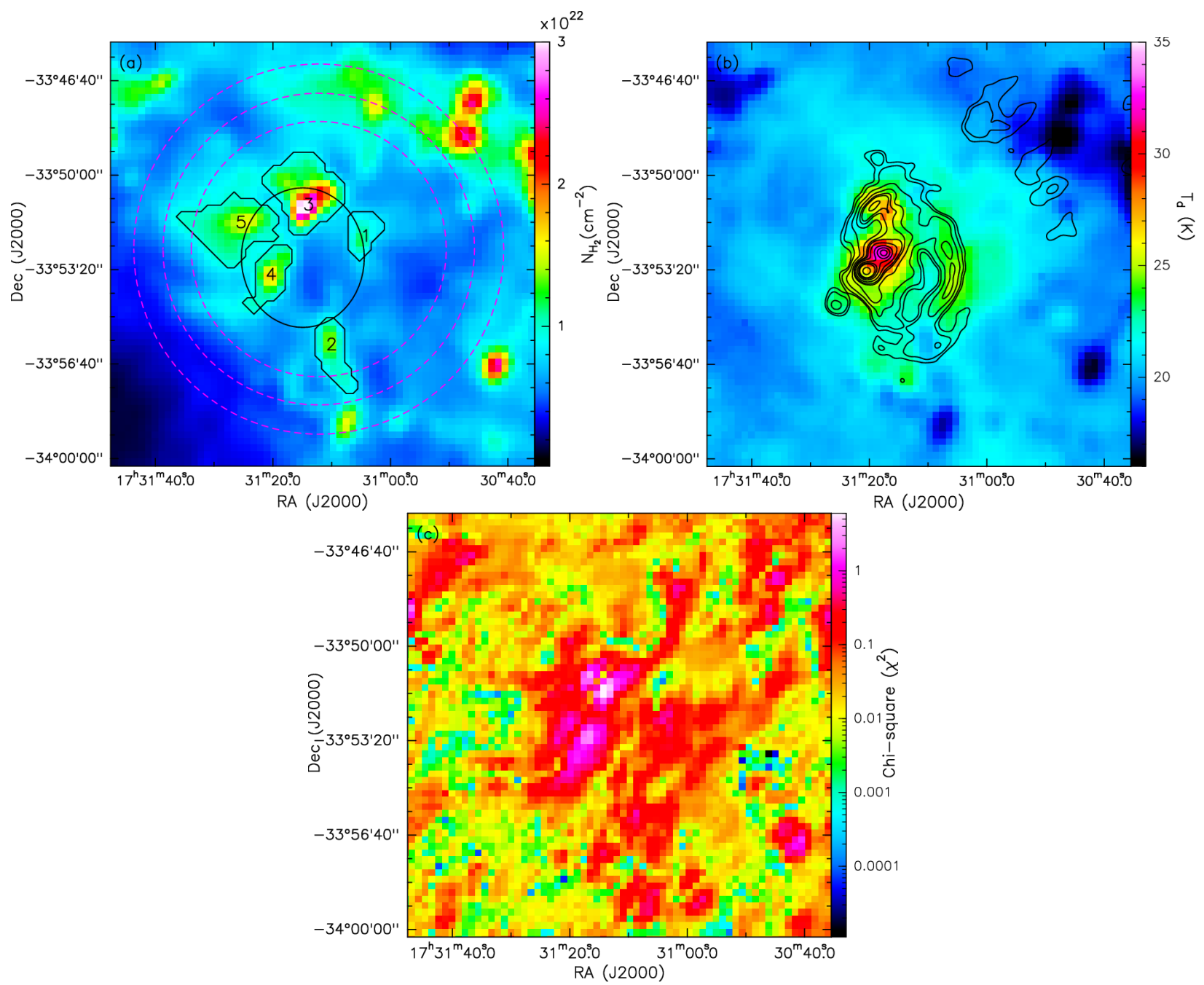

Figure 5. Column density (a) and dust temperature (b) and chi-square (c) maps generated using Herschel FIR data are shown. In plot (a), the black ellipse shows the extent of the bubble and the five identified cold dust clumps are shown as black contours. This plot also shows three concentric circles (dashed magenta) which are used in the study of column density PDFs (see Section 4.2). 610-MHz radio contours are overlaid in plot (b) to correlate the dust temperature with the ionized gas distribution. The contour levels are same as in Figure 3

Table 6. Physical parameters of the clumps associated with CS51. The peak position, radius, mean dust temperature, mean column density, mass and volume density for each clump is listed.

\begin{tabular}{|c|c|c|c|c|c|c|c|c|}
\hline Clump No. & $\begin{array}{c}\text { RA }(2000) \\
\left(\begin{array}{lll}h & m & s\end{array}\right)\end{array}$ & $\begin{array}{c}\mathrm{DEC} \\
(2000) \\
\left(\begin{array}{ccc}0 & \prime\end{array}\right)\end{array}$ & $\begin{array}{l}\text { Radius } \\
\text { (pc) }\end{array}$ & $\begin{array}{l}\text { Mean } \mathrm{T}_{\mathrm{d}} \\
(\mathrm{K})\end{array}$ & $\begin{array}{l}\left.\text { Mean N( } \mathrm{H}_{2}\right) \\
\left(\times 10^{21} \mathrm{~cm}^{-2}\right)\end{array}$ & $\sum_{\left(\times 10^{23} \mathrm{~cm}^{-2}\right)} \mathrm{N}\left(\mathrm{H}_{2}\right)$ & $\begin{array}{l}\text { Mass } \\
\left(\mathrm{M}_{\odot}\right)\end{array}$ & $\begin{array}{c}\mathrm{n}_{\mathrm{H}_{2}} \\
\left(\times 10^{3} \mathrm{~cm}^{-3}\right)\end{array}$ \\
\hline 1 & 173105.27 & -335225.35 & 1.0 & 22.7 & 9.4 & 2.3 & 810 & 2.7 \\
\hline 2 & 173109.68 & -335554.26 & 1.2 & 20.7 & 10.5 & 3.8 & 1340 & 2.5 \\
\hline 3 & 173115.29 & -335115.10 & 1.9 & 22.9 & 18.4 & 13.3 & 4600 & 2.2 \\
\hline 4 & 173119.75 & -335335.20 & 1.2 & 26.8 & 10.1 & 4.2 & 1460 & 2.9 \\
\hline 5 & 173124.48 & -335115.10 & 1.9 & 20.9 & 11.4 & 9.8 & 3420 & 1.7 \\
\hline
\end{tabular}

NIR excess. Sources in ' $\mathrm{T}$ ' region are mainly classical TTauri or Class II stars. Sources in the 'P' region are mostly Class I YSOs. To classify YSOs, we have considered sources falling above T-Tauri locus, since there could be overlap of Class I and Class II sources with Herbig AeBe sources. Following this method we identify 31 YSOs, out of which 2 are Class I YSOs and 29 are Class II YSOs. Given the distance to CS51, the 2MASS photometry would be severely affected foreground interstellar extinction thus increasing the contamination from foreground field stars.

Compiling the YSOs identified in the four methods, we have 17, 70, and 6 Class I, Class II, and Class I/II sources, respectively in the region of $5^{\prime}$ radius centered on CS51. For 

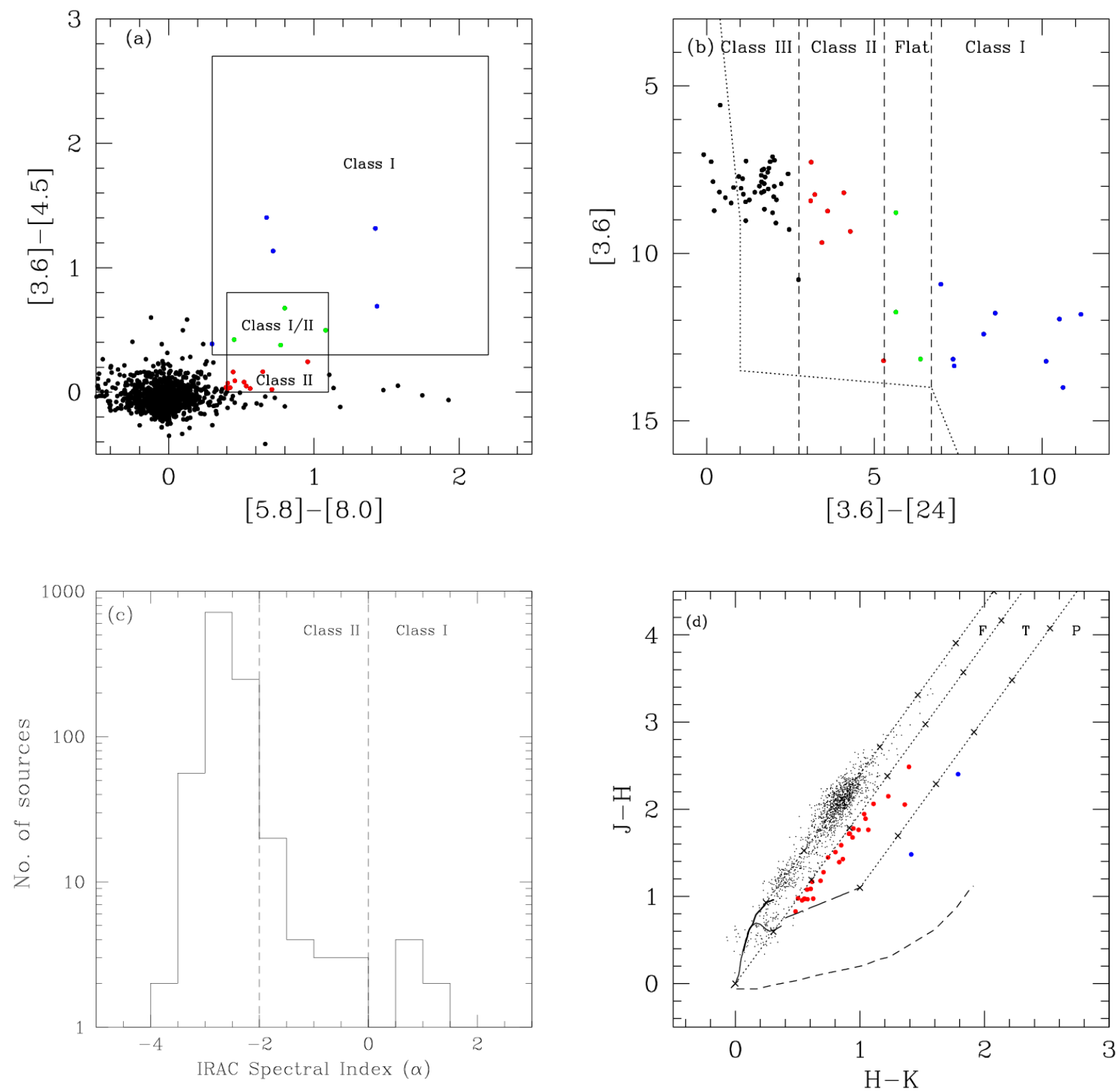

Figure 6. (a) IRAC CCP plot following the method of Allen et al. (2004). The boxes on the plot, adapted from Vig et al. (2007) shows the region for YSOs of different evolutionary classes. (b) [3.6] - [24] vs [3.6] CMP on which the vertical lines adopted from Guieu et al. (2010) and Rebull et al. (2011) demarcate the regions for different classes of YSOs. (c) Histogram plot of the variation of number of sources with IRAC spectral index. The regions for Class I and Class II sources labeled on the plot are adapted from Chavarría et al. (2008). (d) 2MASS J-H vs H-K CCP , in which the three separate regions are labeled as ' $\mathrm{F}$ ', ' $\mathrm{T}$ ', and ' $\mathrm{P}$ ' (refer text). The colour coding for YSOs are as follows: Class I - blue, Class II - red and Class I/II - green.

YSOs identified in more than one scheme, methods (a), (b), (c), and (d) are given preference in this order to assign their class. Figure 7 shows the distribution of the identified YSOs on the $8-\mu \mathrm{m}$ image and Table A1 lists the coordinates, NIR and MIR magnitudes and classification. The spatial distribution of YSOs do not show any particular pattern except a dearth in the bubble interior and an overdensity of Class I YSOs towards the northern periphery of the bubble. Two Class II YSOs are placed in projection towards the central clump thus hinting at a more evolved region compared to the clump towards the northern rim. Without spectroscopic confirmation it is difficult to conclude on the physical association of the identified YSOs with CS51.

\subsection{Molecular line emission towards CS51}

In order to investigate the properties of the clumps associated with the bubble, we have used molecular line data from the MALT90 survey. The available data covers two of the Herschel clumps (\# 3 and \# 4). Study of these molecular 


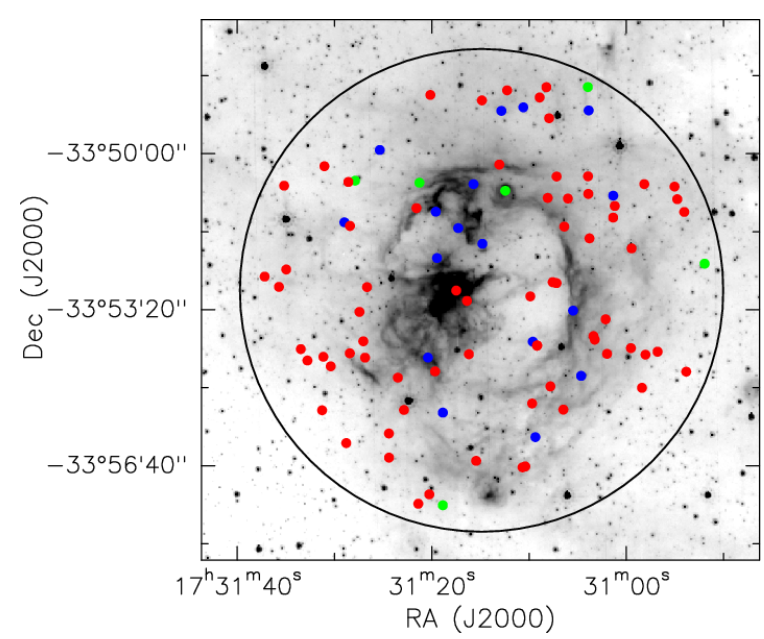

Figure 7. Gray scale is $8.0-\mu \mathrm{m}$ image on which the distribution of YSOs are shown. The colour coding for YSOs are as follows: Class I - blue, Class II - red and Class I/II - green.

lines will enable us to probe the physical, chemical properties and throw light on the evolutionary states of these dense star-forming clumps. Of the 16 molecular-line transitions covered in the MALT90 survey, only five molecular species $\left(\mathrm{C}_{2} \mathrm{H}, \mathrm{HCN}, \mathrm{HCO}^{+}, \mathrm{HNC}\right.$, and $\left.\mathrm{N}_{2} \mathrm{H}^{+}\right)$are detected towards these two clumps. The details of the transitions taken from Foster et al. (2011) and Miettinen (2014) are listed in Table 7. Both the papers, especially the latter, give a nice review on the environment in which these molecules exist and the physical conditions required for their formation.

Figure 8 shows the observed line-spectra at the peak positions for the two clumps. We use the hyperfine structure ( $\mathrm{hfs}$ ) method of CLASS90 to fit the observed spectra for $\mathrm{C}_{2} \mathrm{H}, \mathrm{HCN}, \mathrm{HNC}$, and $\mathrm{N}_{2} \mathrm{H}^{+}$transitions and retrieve the line parameters. As described in Table 7 the molecule $\mathrm{HCO}^{+}$has no hyperfine structure thus a single Gaussian profile was used to fit the spectra. Figure 8 also shows the hfs and Gaussian (for $\mathrm{HCO}^{+}$) fits for the detected transitions. The positions of the hyperfine components are marked in the plots. The retrieved parameters are peak velocity $\left(\mathrm{V}_{\mathrm{LSR}}\right)$, width of velocity $(\Delta V)$, main beam temperature $\left(\mathrm{T}_{\mathrm{mb}}\right)$, and velocity integrated intensity $\left(\int \mathrm{T}_{\mathrm{mb}} \mathrm{dV}\right)$, which are listed in Table 8 Beam correction is applied to the antenna temperature to obtain the main beam temperature, $\mathrm{T}_{\mathrm{mb}}=\mathrm{T}_{\mathrm{A}} / \eta_{\mathrm{mb}}$ (Rathborne et al. 2014) with an assumed value of $\eta_{m b}=0.49$ (Ladd et al. 2005). As revealed in the figure, the signal-to-noise ratio of the observed spectra are less than optimal with several hyperfine components being marginally detected and few others fitting to appreciably large widths (see $\mathrm{C}_{2} \mathrm{H}$ profile for Clump 3 and $\mathrm{HCN}$ profile for Clump 4). In view of this, it should be noted that the retrieved parameters would have significant uncertainties associated. The 0th moment (velocity integrated) contours of two molecules, $\mathrm{HNC}$ and $\mathrm{N}_{2} \mathrm{H}^{+}$, are shown in Figure 9 overlaid on the Spitzer $8 \mu \mathrm{m}$ image. The maps of the other molecules have poor signal-to-noise ratio and hence are not presented. Both molecules show extended emission towards Clump 3 as compared to Clump 4. The plots also show the $610-\mathrm{MHz}$ contours that enable us to correlate the emission from molecular and ionized gas.
We have used RADEX (van der Tak et al. 2007), a onedimensional non-LTE radiative transfer code, to compute the column density of the detected molecular transitions. The input parameters to RADEX are the peak main beam temperature, back ground temperature (2.73 K from Purcell et al. (2006); Yu \& Wang (2015)), kinetic temperature, line width and $\mathrm{H}_{2}$ volume density. We assume the kinetic temperature to be same as dust temperature (Sanhueza et al. 2012 Yu \& Xu 2016 Liu et al. 2016). The $\mathrm{H}_{2}$ volume density and dust temperature values are taken from Section 3.4 which are estimated using the Herschel maps. Using the mean $\mathrm{N}\left(\mathrm{H}_{2}\right)$ for the clumps (listed in Table 6), we estimate the fractional abundances $(x)$ of the detected molecules. The derived column densities and fractional abundances are listed in Table 8. The values estimated for Clumps \#3 and \#4 are in the range derived for a large sample of clumps associated with IRDCs in the work by Miettinen (2014). Similar comparison holds with the results from Vasyunina et al. (2011).

From Figure 9 it is evident that $\mathrm{N}_{2} \mathrm{H}^{+}$and $\mathrm{HNC}$ molecular line emission is appreciably more extended towards Clump 3 in comparison with Clump 4. This is supported by the derived column density values which shows a clear decrease towards Clump 4. Further for $\mathrm{N}_{2} \mathrm{H}^{+}$the fractional abundances and the integrated line intensities also decrease by more than a factor of 4 towards the central Clump 4 . These indicate that Clump 4 is possibly at a later evolutionary stage as compared Clump 3 (Miettinen 2014, Yu \& Wang 2015. Yu \& Xu 2016). The radio peaks A and B fall within Clump 4 with B being close to the peak in the Herschel column density map. Radio peak $\mathrm{C}$ is located in Clump 3. The estimated $\mathrm{C}_{2} \mathrm{H}$ and $\mathrm{N}_{2} \mathrm{H}^{+}$abundances show a decreasing trend with the increasing Lyman continuum photon flux of the clumps further suggesting an earlier evolutionary stage for Clump 3 (Yu \& Xu|2016, Yu \& Wang 2015). Parameters derived from the other detected molecular transitions are fairly consistent with the above picture of evolutionary stage. However, it should be noted here that better signal-to-noise ratio spectral observations are required before conclusively ascertaining the evolutionary stages of the two clumps.

\section{FEEDBACK OF HIGH-MASS STARS AND ORIGIN OF CS51}

Massive stars can influence the parental cloud via various feedback mechanisms. In this section we attempt to understand this in connection with the origin of the bubble CS51 and possible triggered star formation.

\subsection{IRAC band ratio images}

The general bubble structure is a PDR visible at 5.8 and $8 \mu \mathrm{m}$ and an evacuated cavity within this (Churchwell et al. 2006, 2007, Watson et al. 2008, 2009, Anderson et al. 2010, Zavagno et al. 2010, Deharveng et al. 2010, Kendrew et al. 2012). The 5.8 and $8-\mu \mathrm{m}$ emission is attributed to PAH molecules, which are excited by the soft UV photons permeating the PDR, with contribution from thermal emission from dust as well (Watson et al. 2008 Pomarès et al. 2009). 

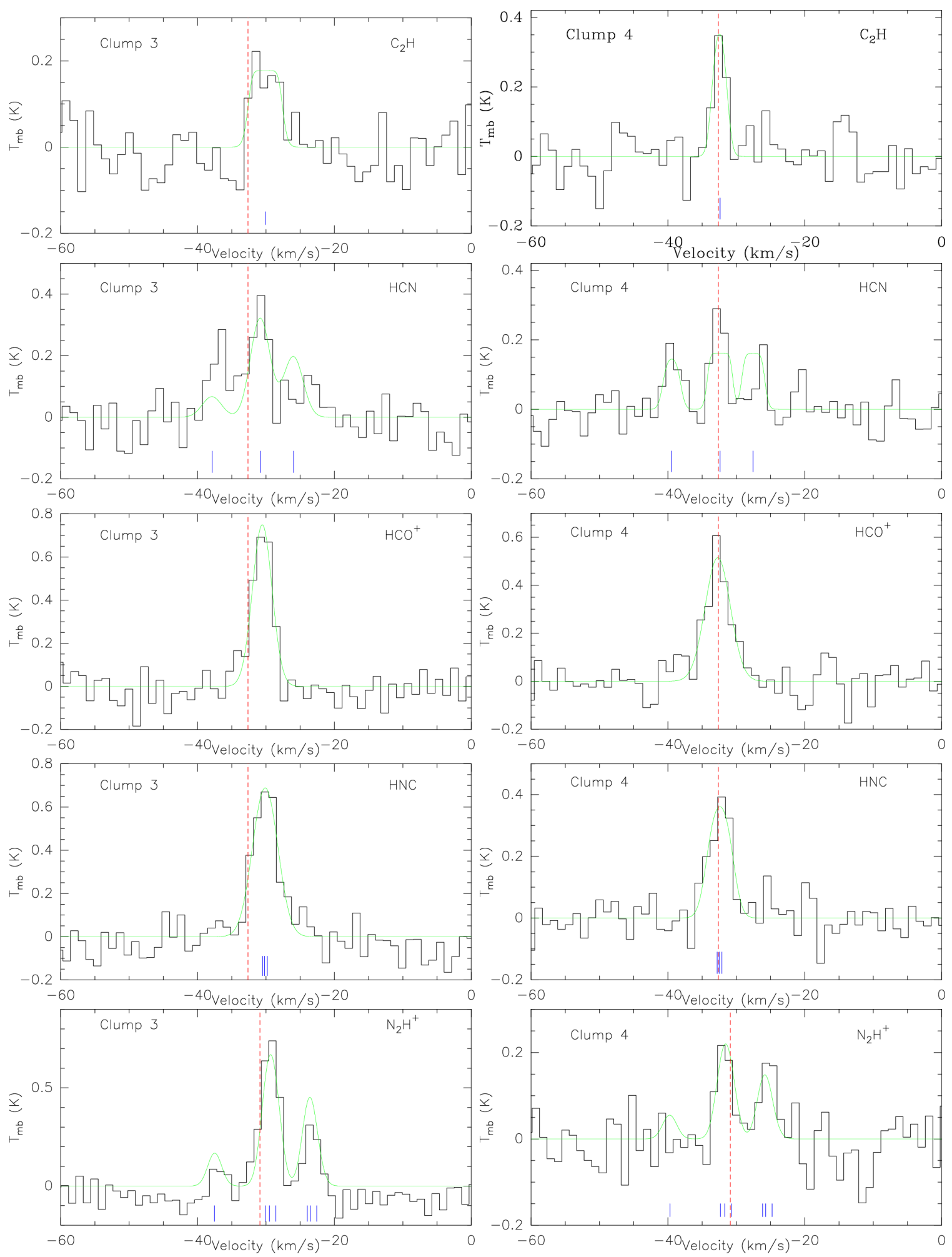

Figure 8. Spectra toward Clumps 3 and Clump 4 associated with CS51. The green curves show the hfs and Gaussian (for $\mathrm{HCO}^{+}$) fits to the spectra. The dashed, vertical red line marks the average peak velocity $\left(\mathrm{V}_{\mathrm{LSR}}\right)$ of all detected molecules of Clump 4 that is used as the systematic velocity. The hyperfine components of the respective molecules are indicated with blue lines. 
Table 7. Summary of detected spectral-line transitions. $E_{u}$ and $n_{\text {crit }}$ are the excitation energy and critical density for the transitions. These are calculated adopting values from the Leiden Atomic and Molecular Database (LAMD; Schöier et al. 2005) and Cologne Database for Molecular Spectroscopy (CDMS; Müller et al. 2001, 2005), assuming a gas temperature of $20 \mathrm{~K}$.

\begin{tabular}{lcccl}
\hline Transition & $\begin{array}{c}\text { Frequency }(\nu) \\
(\mathrm{GHz})\end{array}$ & $\begin{array}{c}E_{u} / k_{B} \\
(\mathrm{~K})\end{array}$ & $\begin{array}{c}n_{\text {crit }} \\
\left(\mathrm{cm}^{-3}\right)\end{array}$ & \multicolumn{1}{c}{ Remarks } \\
\hline $\mathrm{C}_{2} \mathrm{H}(1-0) 3 / 2-1 / 2$ & 87.316925 & 4.19 & $2 \times 10^{5}$ & $\begin{array}{l}\text { Tracer of photodissociation region } \\
\mathrm{N}_{\mathrm{J}}=1_{3 / 2}-0_{1 / 2} \text { is split into three hf components } \\
\text { tracer of high column density, optical depth }\end{array}$ \\
$\mathrm{HCN}(1-0)$ & 88.631847 & 4.25 & $3 \times 10^{6}$ & $\begin{array}{l}\mathrm{J}=1-0 \text { is split into three hf components } \\
\text { High column density, kinematics }\end{array}$ \\
$\mathrm{HCO}^{+}(1-0)$ & 89.188526 & 4.28 & $2 \times 10^{5}$ & $\begin{array}{l}\text { High column density, cold gas tracer } \\
\text { HNC }(1-0)\end{array}$ \\
90.663572 & 4.35 & $3 \times 10^{5}$ & $\begin{array}{l}\text { Three hf components } \\
\text { High column density, depletion resistant, optical depth } \\
\mathrm{J}=1-0 \text { line has 15 hf components out of which seven have a different frequency }\end{array}$ \\
\hline
\end{tabular}

Table 8. Parameters of molecular transitions detected towards the two dust clumps. The LSR velocity $\left(\mathrm{V}_{\mathrm{LSR}}\right)$, line width $(\Delta \mathrm{V})$, mainbeam temperature $\left(\mathrm{T}_{\mathrm{mb}}\right)$, and velocity integrated intensity $\left(\int \mathrm{T}_{\mathrm{mb}} \mathrm{dV}\right)$ are obtained from the hfs fitting method of CLASS90. The column density $(N)$ values are estimated using RADEX and the fractional abundances $(x)$ are derived using mean $\mathrm{H}_{2}$ column density of $1.8 \times 10^{22} \mathrm{~cm}^{-2}$ and $1.0 \times 10^{22} \mathrm{~cm}^{-2}$ for Clump 3 and Clump 4, respectively (refer Section 3.4 .

\begin{tabular}{lcccccc}
\hline Transition & $\begin{array}{c}\mathrm{V}_{\mathrm{LSR}} \\
\left(\mathrm{km} \mathrm{s}^{-1}\right)\end{array}$ & $\begin{array}{c}\Delta \mathrm{V} \\
\left(\mathrm{km} \mathrm{s}^{-1}\right)\end{array}$ & $\begin{array}{c}\mathrm{T}_{\mathrm{mb}} \\
(\mathrm{K})\end{array}$ & $\begin{array}{c}\int \mathrm{T}_{\mathrm{mb}} \mathrm{dV} \\
\left.(\mathrm{K} \mathrm{km} \mathrm{s})^{-1}\right)\end{array}$ & $\begin{array}{c}N \\
\left(\times 10^{14} \mathrm{~cm}^{-2}\right)\end{array}$ & $\begin{array}{c}x \\
\left(10^{-9}\right)\end{array}$ \\
\hline $\mathrm{C}_{2} \mathrm{H}$ & -30.10 & 2.61 & 0.27 & 0.33 & 3.59 & 19.90 \\
$\mathrm{HCN}$ & -30.80 & 3.31 & 0.38 & 1.25 & 6.05 & 33.63 \\
$\mathrm{HCO}^{+}$ & -30.54 & 3.36 & 0.75 & 2.69 & 0.52 & 2.89 \\
$\mathrm{HNC}^{\mathrm{N}}$ & -30.20 & 4.18 & 0.69 & 3.18 & 2.62 & 14.57 \\
$\mathrm{~N}_{2} \mathrm{H}^{+}$ & -29.50 & 2.38 & 0.77 & 2.46 & 3.11 & 17.29 \\
\hline & & & Clump 3 & & \\
\hline $\mathrm{C}_{2} \mathrm{H}$ & -32.40 & 1.96 & 0.36 & 0.86 & 2.47 & 23.97 \\
$\mathrm{HCN}$ & -32.40 & 1.90 & 0.31 & 0.76 & 1.71 & 16.64 \\
$\mathrm{HCO}+$ & -32.71 & 4.33 & 0.52 & 2.38 & 0.28 & 2.75 \\
$\mathrm{HNC}^{+}$ & -32.50 & 3.18 & 0.38 & 1.50 & 0.69 & 6.70 \\
$\mathrm{~N}_{2} \mathrm{H}^{+}$ & -31.70 & 2.41 & 0.23 & 0.61 & 0.45 & 4.35 \\
\hline
\end{tabular}

In their detailed study on M17, Povich et al. (2007), proposed the use of IRAC band ratio images to understand the interaction of massive stars with their surrounding and delineate regions of PAH destruction. They further confirmed the disappearance of PAH in the destruction zone from spectroscopic data. Following this pioneering work, Watson et al. (2008) used this technique in several bubbles to locate the PDR around them. This technique exploits the fact that three of the four IRAC bands (at 3.6, 5.8, $8.0 \mu \mathrm{m}$ ) sample several PAH emission features (see Table 5 of Povich et al. 2007) whereas the $4.5-\mu \mathrm{m}$ band is PAH-free.

We adopt the same procedure described in Povich et al. (2007) of using residual images (after removing point sources) followed by median filtering and smoothening before taking the ratios. Figure 10, shows the ratio images. Figure 10 (a) and (b) show the ratio of $8.0-\mu \mathrm{m}$ and $5.8-\mu \mathrm{m}$ images, respectively with the PAH-free $4.5-\mu \mathrm{m}$ image. The bright emission (seen as dark in the colour inverted plots) towards the bubble rim in these ratio maps show the location of the PAH zones thus defining the PDR related to CS51. This is similar to the results obtained by Watson et al. (2008) and Dewangan et al. (2012). However, it should be noted that the ratio maps also show enhanced emission towards the likely centre of the bubble coinciding with the region of bright radio emission. Given that PAH would be destroyed in the harsh radiation field close to the ionizing star (Watson et al. (2008); Deharveng et al. (2010) and references therein), this bright region could possibly be from the thermal dust continuum which is supported by the bright $24-\mu \mathrm{m}$ emission that arises mostly near the hot star when the dust is heated to $\sim 100 \mathrm{~K}$. Figure 10 (c) shows the ratio map between two PAH bearing bands $(8.0 \mu \mathrm{m} / 5.8 \mu \mathrm{m})$. As expected, the rim emission is invisible here. It is to be noted that while these ratio images give the overall picture of PAH emission in the PDR, it should be kept in mind that the contribution from thermal dust and other atomic and molecular features would also be present. Thus these ratio maps should be taken as illustrative unless confirmed with spectroscopy. Nevertheless, the ratio maps do confirm with the generally accepted picture of bubbles. 

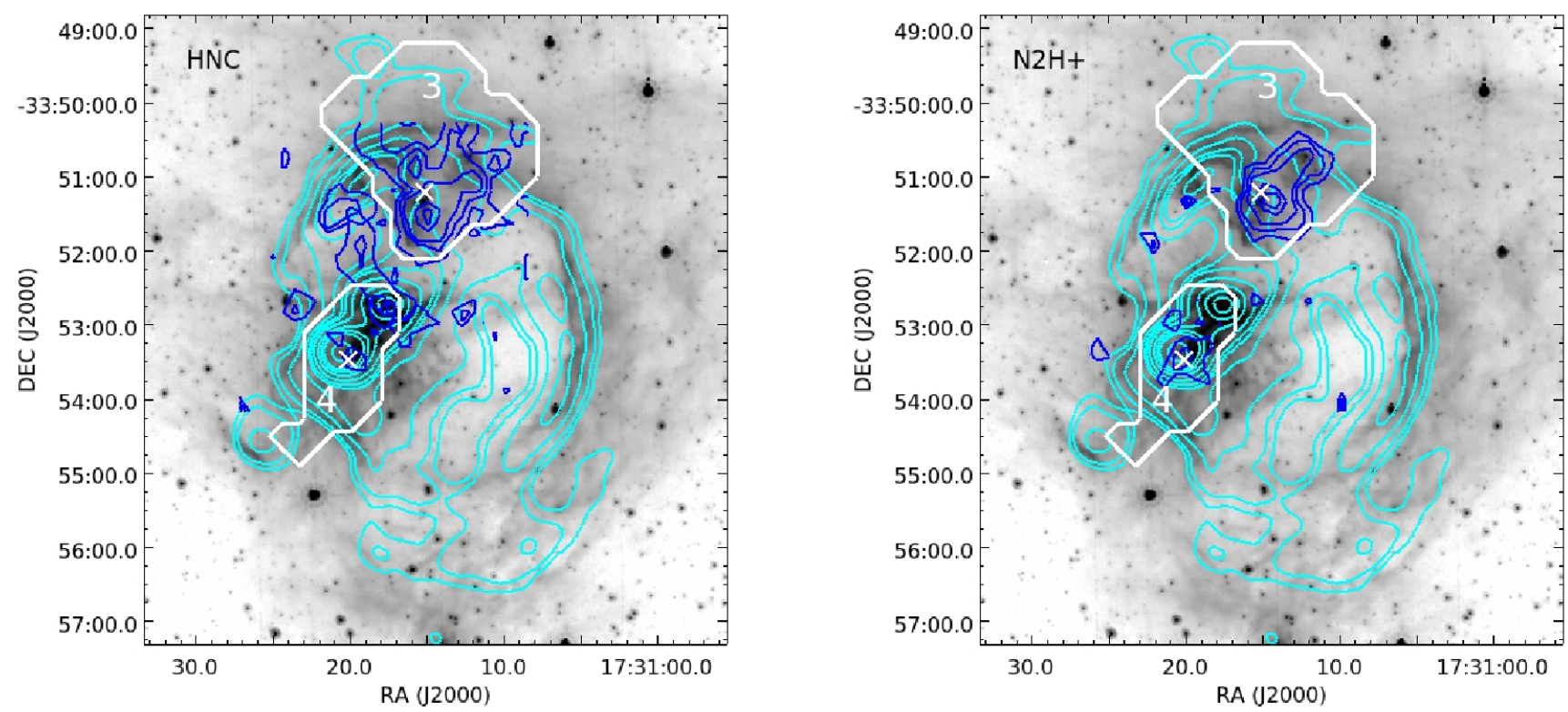

Figure 9. Spitzer $8-\mu \mathrm{m}$ is shown in gray scale. Blue contours are the integrated intensity maps. The contour levels starts from $3 \sigma$, where $\sigma=0.4 \mathrm{~K} \mathrm{~km} \mathrm{~s}^{-1}$ for both the molecules. $610-\mathrm{MHz}$ radio contours in cyan shows the distribution of ionized gas. The contour levels are same as in Figure 3 The white crosses are the pointing of MALT90 observation. The retrieved clump apertures (see section 3.4 are also shown.
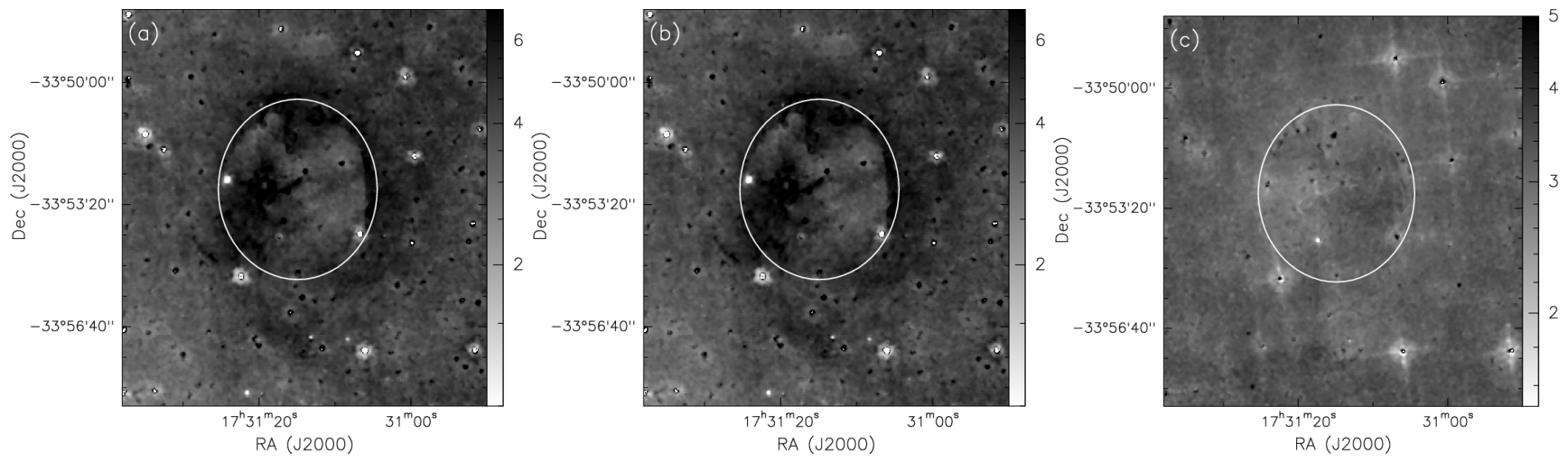

Figure 10. IRAC ratio maps $8.0 \mu \mathrm{m} / 4.5 \mu \mathrm{m}$ (a), $5.8 \mu \mathrm{m} / 4.5 \mu \mathrm{m}$ (b) and $8.0 \mu \mathrm{m} / 5.8 \mu \mathrm{m}$ (c) of the region associated to bubble CS51. The bright emission in (a) and (b) show the PAH emission regions. The extent of the bubble is shown as a black ellipse. Point source removal at $4.5 \mu \mathrm{m}$ is not very good since the region is densely populated. The residuals show up in the ratio images (a) and (b).

\subsection{Column density probability distribution function}

Structures in the form of clumps or condensations at the border of IR dust bubbles, pillars and protrusions pointing towards the ionizing source have been observed in several studies (Pomarès et al. 2009, Petriella, Paron \& Giacani 2010, Zavagno et al. 2010, Anderson et al. 2012, Ji et al. 2012 Liu et al. 2015, 2016). The formation of these structures and the star formation therein are discussed invoking the 'collect and collapse' (CC) model (Elmegreen \& Lada 1977) or the 'radiation driven implosion' (RDI) model (Bertoldi 1989 Lefloch \& Lazareff 1994), thus probing the feasibility of triggered star formation in the 'accumulated' versus 'pre-existing' scenarios.

Understanding the role of density compression of ionized gas into a molecular cloud and that of ram pressure of turbulence of the cloud are crucial aspects in deciphering the impact of high-mass stars and this is discussed in detail by Tremblin et al. (2012a b, 2014). They use the probability distribution function (PDF) of the column density around the ionized regions to study the feedback of the massive star. Their studies show that the column density PDF displays a single peak if turbulence dominates and the distribution becomes bimodal with a second peak forming at higher densities if the ionization pressure becomes larger than the turbulence. Liu et al. (2016, 2017a) have implemented this technique on two HiI regions / bubbles. Adopting the same formulation, we investigate the nature of the column density PDFs in the region associated with CS51. We generate the PDF for the regions encircled within the three circles shown in Figure 5(a). These concentric circles are constructed with a separation of $1^{\prime}$ and for clarity we name them as Circle 1, 2 , and 3 in increasing order of radius. The functional form for the bimodal PDF used to fit the column density is given 
by the following expression (Liu et al. 2016, Schneider et al. 2012, Tremblin et al. 2014)

$p(\eta)=\frac{p_{0}}{\sqrt{2 \pi \sigma_{0}^{2}}} \exp \left(\frac{-\left(\eta-\mu_{0}\right)^{2}}{2 \sigma_{0}^{2}}\right)+\frac{p_{1}}{\sqrt{2 \pi \sigma_{1}^{2}}} \exp \left(\frac{-\left(\eta-\mu_{1}\right)^{2}}{2 \sigma_{1}^{2}}\right)$

where, $\eta=\ln (N / \bar{N}), N$ is the column density and $\bar{N}$ is the average column density taken over Circle 3. $p_{i}, \mu_{i}$, and $\sigma_{i}$ are the integral, mean, and dispersion of each component. In the above expression, the first lognormal component at low column density is associated with the initial turbulent molecular cloud and the second component at high column density is attributed to compression by the ionized gas pressure (Liu et al. 2016, Tremblin et al. 2014).

Figure 11 shows the column density PDFs in the three identified circles covering the ionized emission associated with CS51 and the surrounding cloud. The fitted parameters are listed in Table 9. The PDFs fit fairly well and all of them clearly show the second peak consistent with the results obtained for the bubbles N4, RCW 79 and RCW 120 (Tremblin et al. 2014, Liu et al. 2016, 2017a). Similar trend of $p_{0}$ increasing and $p_{1}$ decreasing as we move outwards is seen for CS51. As noted by Liu et al. (2016) and discussed by Tremblin et al. (2014), the decrease in $p_{1}$ with increasing radius indicates decreasing effect of compression due to ionized gas for larger regions. The results obtained reiterates the strong influence of the expanding bubble on its immediate surrounding. The second lognormal form suggesting compression from ionized gas would likely account for the condition required for triggered star formation. The high column density region shows signature of a power-law tail, which is generally attributed to on-going star formation (Schneider et al. 2015, 2012, Liu et al. 2017a; Schneider et al. 2013 (Russeil et al. 2013). Insufficient data points and low signal-to-noise ratio prevents us from fitting a powerlaw and attempting a more quantitative comparison with the above studies.

\subsection{Collect and collapse scenario}

The previous sub-section showed the role of ionization compression in CS51. The effect of this in triggered star formation has been in focus since the pioneering work by Elmegreen \& Lada (1977). As mentioned earlier, several studies have focused on the mechanism behind this triggering process - CC or RDI. The detection of five dense clumps in the PDR region suggest that the shocked molecular layer is mostly swept up and accumulated during the bubble expansion. Thus we explore the possibility of the $\mathrm{CC}$ model at work around CS51. To examine this, we estimate the dynamical age of the bubble and compare the same with the fragmentation time of the surrounding cloud.

Assuming that the HiI region expands in a homogeneous molecular cloud, the dynamical age of the HiI region is estimated from the following well known expressions (Spitzer 1978, Dyson \& Williams 1980)

$$
\mathrm{R}_{\mathrm{st}}=\left[\frac{3 \mathrm{~N}_{\mathrm{Lyc}}}{4 \pi \mathrm{n}_{0}^{2} \alpha_{\mathrm{B}}}\right]^{1 / 3}
$$
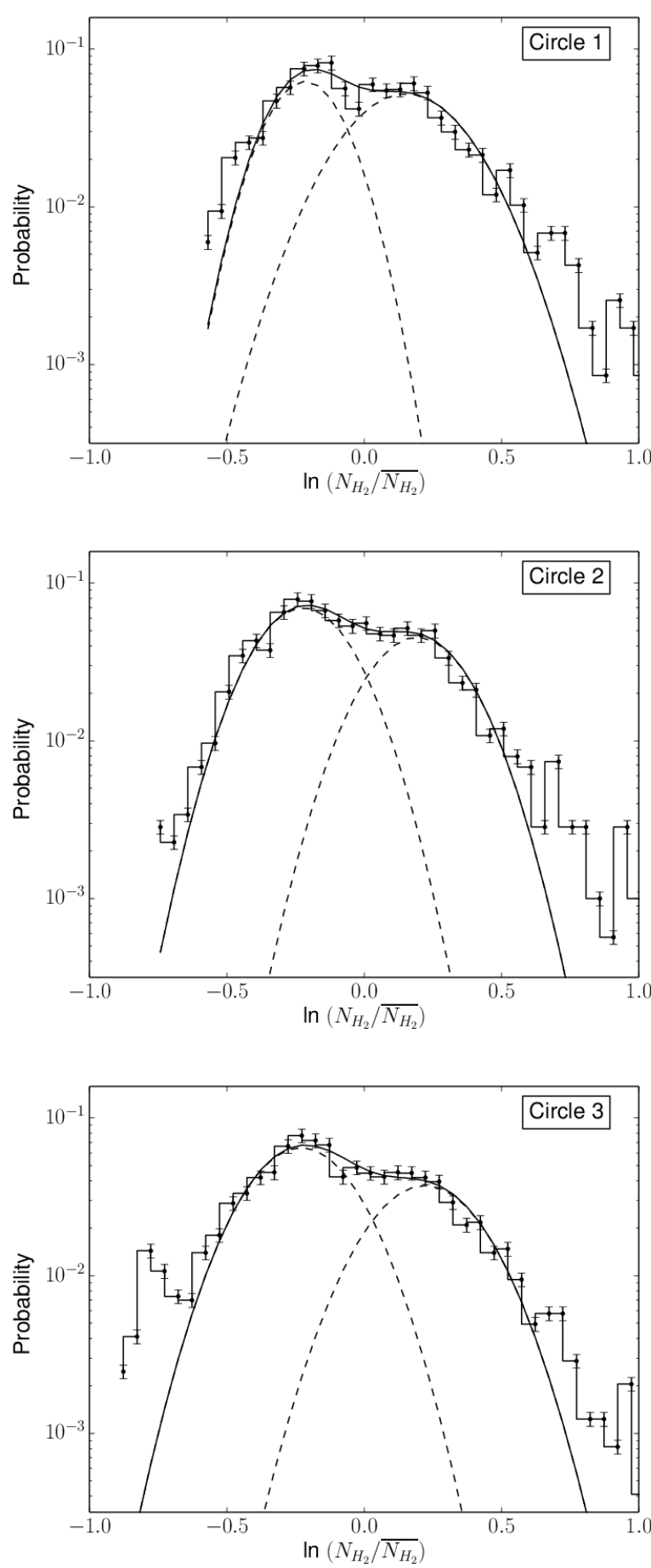

Figure 11. Plots of column density PDFs over the three circular regions. The dashed lines are the two lognormal components and the best fit (black solid line) is the sum of these two. $\overline{N_{H_{2}}}$ is the mean column density computed over the largest region.

$$
\mathrm{t}_{\mathrm{dyn}}=\frac{4}{7} \frac{\mathrm{R}_{\mathrm{st}}}{\mathrm{C}_{\mathrm{Hii}}}\left[\left(\frac{\mathrm{R}_{\mathrm{if}}}{\mathrm{R}_{\mathrm{st}}}\right)^{7 / 4}-1\right]
$$

where, $R_{\text {st }}$ is the Strömgren radius, $N_{L y c}$ is the Lyman continuum photons flux responsible for the HII region and $n_{0}$ is 
Infrared dust bubble CS51

Table 9. Retrieved parameters from the lognormal fit to column density PDFs.

\begin{tabular}{cccccccc}
\hline Circle & radius $\left({ }^{\prime}\right)$ & $p_{0}$ & $\mu_{0}$ & $\sigma_{0}$ & $p_{1}$ & $\mu_{1}$ & $\sigma_{1}$ \\
\hline 1 & 4.5 & 0.020 & -0.215 & 0.131 & 0.027 & 0.152 & 0.206 \\
2 & 5.5 & 0.028 & -0.222 & 0.163 & 0.019 & 0.193 & 0.171 \\
3 & 6.5 & 0.029 & -0.229 & 0.179 & 0.018 & 0.222 & 0.190 \\
\hline
\end{tabular}

the initial particle density of the ambient gas. $\alpha_{\mathrm{B}}$ is the coefficient of radiative recombination and it is calculated from Kwan (1997), to be $2.6 \times 10^{-13}\left(10^{4} \mathrm{~K} / \mathrm{T}\right)^{0.7} \mathrm{~cm}^{3} \mathrm{sec}^{-1}$. $\mathrm{t}_{\mathrm{dyn}}$ is the dynamical age of the HiI region, $\mathrm{C}_{\mathrm{Hii}}$ is the isothermal sound speed (assumed to be $10 \mathrm{~km} \mathrm{~s}^{-1}$ ), $\mathrm{R}_{\mathrm{if}}$ is the radius of HiI region which is approximately taken as the radius of the bubble $\left(2.1^{\prime}\right)$. To estimate $\mathrm{n}_{0}$, we take the total mass of the swept up molecular shell which is the sum of the masses of the five detected clumps on the bubble periphery $\left(11630 \mathrm{M}_{\odot}\right)$ and assume that it was distributed homogeneously within the bubble radius. To this we add the mass of the ionized component $\left(300 \mathrm{M}_{\odot}\right)$. This gives an estimate of $\mathrm{n}_{0}=2.4 \times 10^{3} \mathrm{~cm}^{-3}$ which defines a lower limit since it does not take into account the ionized gas that probably would have escaped through the rupture of bubble or absorbed by dust. From the estimated parameters of the clumps (see Table 6), we get an average number density of $\mathrm{n}_{0}=4.8 \times 10^{3} \mathrm{~cm}^{-3}$ which can be considered as the upper limit. Using the above values, we estimate the dynamical age of CS51 to be $0.9-1.3$ Myr. This estimate should be taken with caution since the assumption of expansion in a uniform medium is not realistic.

We proceed further to investigate fragmentation of the collected shell material using the model of Whitworth et al. (1994). They give the following expression to estimate the fragmentation time.

$$
\mathrm{t}_{\text {frag }}=1.56 \mathrm{a}_{\mathrm{s}}^{7 / 11} \mathrm{~N}_{49}^{-1 / 11} \mathrm{n}_{3}^{-5 / 11} \mathrm{Myr}
$$

where, $a_{s}$ is the sound speed in the shocked layer (the turbulent velocity) in units of $0.2 \mathrm{~km} \mathrm{sec}^{-1}, \mathrm{~N}_{49}$ is the ionizing photon in units of $10^{49}$ photons $\mathrm{sec}^{-1}$, and $\mathrm{n}_{3}$ is the initial particle density of the ambient gas $\left(\mathrm{n}_{0}\right)$ in units of $10^{3} \mathrm{~cm}^{-3}$. Taking $a_{s}$ to be $0.3 \mathrm{~km} \mathrm{~s}^{-1}$ at the derived dust temperatures, we estimate the fragmentation time, $\mathrm{t}_{\text {frag }}$, to be $0.5 \mathrm{Myr}$ for $\mathrm{n}_{0}=2.4 \times 10^{3} \mathrm{~cm}^{-3}$ and 0.4 Myr for $\mathrm{n}_{0}=4.8 \times 10^{3} \mathrm{~cm}^{-3}$. The estimated fragmentation time is shorter (by a factor of 2) than the dynamical age of the HiI region implying that the shell of collected material has enough time to gravitationally fragment during the expansion of the bubble. Similar results are obtained for N4, G8.14+0.23 and G24.136+00.436, where the fragmentation time is less than the dynamical time (Liu et al. 2016, 2015. Dewangan et al. 2012). In contrast, Ji et al. (2012) derive fragmentation time significantly larger than the dynamical age for bubble N22 and they discard the CC process. The above estimates of the timescales involved support the CC process as the likely mechanism for the formation of the identified clumps and the associated star formation. It should however be kept in mind that, this simple model does not conclusively exclude the RDI process and thus higher resolution observations are required to probe the dense cores within these clumps and compare their masses, radii and separation with the theoretical predictions of the CC mechanism (Whitworth et al. 1994; Liu et al. 2017b).

\subsection{What do the clumps reveal?}

As discussed in Section 3.6 the Clumps \#3 and \#4 show trends of different evolutionary stages with Clump 4 being more evolved. Association with radio peaks and $24 \mu \mathrm{m}$ emission indicate that these are active star forming clumps. Further, the NIR and MIR colours have shown the presence of YSOs likely to be associated with the clumps with an overdensity of Class I YSOs seen towards Clump 3.

From the derived centroid velocities of the molecular transitions in the clumps, it is seen that the lines related to Clump 3 are red-shifted by $\sim 2 \mathrm{~km} \mathrm{~s}^{-1}$ with respect to a systematic velocity of $-32.34 \mathrm{~km} \mathrm{~s}^{-1}$ which is taken as the average of velocities of detected molecular line emission in Clump 4. Clump 4 is located within the bubble (and closer to the centre) correlating well with the likely location of the exciting $\operatorname{star}(\mathrm{s})$. The above is consistent with the picture of the formation of CS51 where the shell of dust and gas seen is presumably swept up by the expanding HiI region. Hence Clump 3 that is located towards the northern rim is expected to be moving away from the central clump indicating the expansion of the bubble. Similar expansion is revealed from the molecular line data of bubble N4 where the clumps towards the south-east and north-west part of N4 are seen to have blue-shifted and red-shifted velocities, respectively compared to a systematic velocity. It should be noted here that we do not have molecular line data for Clumps \#1, \#2, and \# 5 to strengthen the picture of expansion.

From the mass and size estimates of the five clumps discussed in Section 3.4. Clumps 2, 3, 4, and 5 qualify as potential high-mass forming ones as they satisfy the threshold criteria of $\mathrm{m}(\mathrm{r}) \geqslant 870 \mathrm{M}_{\odot}(\mathrm{r} / \mathrm{pc})^{1.33}$, where $\mathrm{m}(\mathrm{r})$ is mass of clump and $\mathrm{r}$ is the effective radius of clump, discussed in Kauffmann \& Pillai (2010). We have also investigated the gravitational state of Clumps \#3 and \#4 by deriving the virial parameter $(\alpha)$ defined as the ratio between virial mass to dust mass. This parameter indicates whether a clump is gravitationally bound or not. Clumps are likely to undergo gravitational collapse if $\alpha<1$ and in the absence of magnetic field can be considered as bound. If the value of $\alpha$ exceeds unity, then in all likelihood expansion is possible unless some external mechanism constrains the cloud. Virial mass of each clump is derived from the following equation (Contreras et al. 2016).

$\mathrm{M}_{\mathrm{vir}}=\frac{5 \mathrm{r} \Delta \mathrm{V}^{2}}{8 \ln (2) \mathrm{a}_{1} \mathrm{a}_{2} \mathrm{G}} \sim 209 \frac{1}{\mathrm{a}_{1} \mathrm{a}_{2}}\left(\frac{\Delta \mathrm{V}}{\mathrm{km} \mathrm{s}^{-1}}\right)^{2}\left(\frac{\mathrm{r}}{\mathrm{pc}}\right) \mathrm{M}_{\odot}$ 
where, the constant $\mathrm{a}_{1}$ is the correction for power-law distribution, which can be expressed as $\mathrm{a}_{1}=(1-\mathrm{p} / 3) /(1-2 \mathrm{p} / 5)$, for $\mathrm{p}<2.5$ (Bertoldi \& McKee 1992). We adopt a power-law density distribution of $p=1.8$ (Contreras et al. 2016). The constant $\mathrm{a}_{2}$ is correction for non-spherical shape. We assume the clumps to be spherical and take $\mathrm{a}_{2}$ as $1 . \Delta \mathrm{V}$ and $\mathrm{r}$ are the line width and radius (listed in Table 6) of the clumps. To derive virial mass and virial parameter, we use the optically thin $\mathrm{N}_{2} \mathrm{H}^{+}$emission because an optically thick line would overestimate the virial mass (Sanhueza et al. 2012). Thus using the above equation and the line width obtained in Section 3.6, we derive virial masses of $\sim 1100$ and $835 \mathrm{M}_{\odot}$ for Clumps 3 and 4, respectively. Taking the ratio with the mean dust masses of the clumps (see Table 6), we estimate the virial parameter to be 0.2 and 0.6 for Clumps 3 and 4 , respectively. These estimates are consistent with the nature of the clumps where signature of active star formation is observed.

\section{SUMMARY}

In this work, we have carried out a detailed multi-wavelength study towards the southern IR bubble CS51 which is associated with IRAS 17279-3350. Our main results are the following.

(i) The associated ionized emission probed at 610 and $1300 \mathrm{MHz}$ displays a complex morphology. The ionized emission mostly fills the bubble interior with a cavity towards the south-west, which is more pronounced at $1300 \mathrm{MHz}$. The observed Lyman continuum photon flux translates to a spectral type of $\mathrm{O} 6 \mathrm{~V}-\mathrm{O} 5.5 \mathrm{~V}$ for the exciting source under the assumption of optically thin, free-free emission. The mass of this associated ionized gas within the bubble is estimated to be $\sim 300 \mathrm{M}_{\odot}$.

(ii) The GMRT radio maps reveal the presence of three components (A, B, and C) associated with CS51. They show up as compact regions of enhanced emission. The component A is located towards the likely center of the bubble, the component B (brightest among them) is towards the southeast periphery and the component $\mathrm{C}$ is located in the northwest rim of the bubble.

(iii) The 610 - $1300 \mathrm{MHz}$ spectral index map shows the coexistence of both thermal free-free emission and non-thermal synchrotron emission. The compact regions $\mathrm{A}$ and $\mathrm{C}$ are seen to be associated with thermal emission and component $\mathrm{B}$ shows signature of non-thermal emission.

(iv) From the optical (B-band) and NIR colours, we identify three sources as potential candidate exciting star(s) from a sample of sources located towards the radio and $24 \mu \mathrm{m}$ emission peaks.

(v) The column density and dust temperature maps are generated by pixel-wise SED modeling of the thermal dust emission using FIR Herschel data. The column density map shows a fragmented shell like structure harbouring four clumps towards the periphery of the bubble and one clump is detected within the bubble close to the likely centre. Dust temperature map shows the presence of warmer dust in the bubble interior consistent with the presence of ionized emission.

(vi) Five molecular transitions $\left(\mathrm{C}_{2} \mathrm{H}, \mathrm{HCN}, \mathrm{HCO}^{+}, \mathrm{HNC}\right.$, and $\mathrm{N}_{2} \mathrm{H}^{+}$) are detected towards two clumps (\#3 and \#4).
The hyperfine components of $\mathrm{C}_{2} \mathrm{H}, \mathrm{HCN}, \mathrm{HNC}$, and $\mathrm{N}_{2} \mathrm{H}^{+}$ are clearly detected above noise level. The velocity integrated (0th moment) map for $\mathrm{N}_{2} \mathrm{H}^{+}$and $\mathrm{HNC}$ are seen to be appreciably extended towards Clump 3. Within the uncertainties due to low signal-to-noise ratio of the observed spectra, the derived line parameters, column density, and fractional abundance suggest a possible earlier evolutionary phase of Clump 3 as compared to Clump 4.

(vii) The IRAC ratio maps involving PAH-bearing and PAH-free bands show the presence of PAH emission associated with the PDR of CS51 thus confirming the generally accepted bubble formation mechanism and related MIR emission.

(viii) The column density PDFs display a bimodal distribution thus demonstrating the strong influence of expanding bubble on its surrounding where compression due to ionized gas pressure dominates the effect of turbulence.

(ix) Assuming the expansion of the associated Hil region to occur in a uniform medium, the dynamical age is estimated to be $0.9-1.3 \mathrm{Myr}$, which is higher than the derived fragmentation time of $0.4-0.5 \mathrm{Myr}$. This indicates that the collect and collapse mechanism is possibly at work around the bubble CS51 and responsible for triggering star formation towards the border of the bubble.

(x) The estimated values of mass and radius of the clumps, that lie between $810-4600 \mathrm{M}_{\odot}$ and $1.0-1.9 \mathrm{pc}$, respectively, indicate all except Clump 1 to be high-mass star forming clumps.

(xi) The centroid velocities of the molecular transitions detected show signatures of expansion of the bubble. The Clump 3 located towards the northern rim is seen to have red-shifted velocities with respect the central Clump 4.

Acknowledgment : We place on record our sincere thanks to the referee for carefully going through the manuscript and giving valuable suggestions. We thank the staff of the GMRT, that made the radio observations possible. GMRT is run by the National Centre for Radio Astrophysics of the Tata Institute of Fundamental Research. This work is based [in part] on observations made with the Spitzer Space Telescope, which is operated by the Jet Propulsion Laboratory, California Institute of Technology under a contract with NASA. This publication made use of data products from Herschel (ESA space observatory) and the Millimetre Astronomy Legacy Team $90 \mathrm{GHz}$ (MALT90) survey.

\section{REFERENCES}

Allen L. E. et al., 2004, ApJS, 154, 363

Anderson L. D. et al., 2012, A\&A, 542, A10

Anderson L. D. et al., 2010, A\&A, 518, L99

André P. et al., 2010, A\&A, 518, L102

Aniano G., Draine B. T., Gordon K. D., Sandstrom K., 2011, PASP, 123, 1218

Arthur S. J., Kurtz S. E., Franco J., Albarrán M. Y., 2004, ApJ, 608, 282

Battersby C. et al., 2011, A\&A, 535, A128

Baug T., Dewangan L. K., Ojha D. K., Ninan J. P., 2016, ApJ, 833, 85

Becker R. H., White R. L., Helfand D. J., Zoonematkermani S., 1994, ApJS, 91, 347

Beckwith S. V. W., Sargent A. I., Chini R. S., Guesten R., 1990, AJ, 99, 924 
Beltrán M. T., Brand J., Cesaroni R., Fontani F., Pezzuto S., Testi L., Molinari S., 2006, A\&A, 447, 221

Benaglia P., Romero G. E., Koribalski B., Pollock A. M. T., 2005, A\&A, 440, 743

Benjamin R. A. et al., 2003, PASP, 115, 953

Bertoldi F., 1989, ApJ, 346, 735

Bertoldi F., McKee C. F., 1992, ApJ, 395, 140

Bessell M. S., Brett J. M., 1988, PASP, 100, 1134

Caswell J. L. et al., 2010, MNRAS, 404, 1029

Caswell J. L., Haynes R. F., 1987, A\&A, 171, 261

Chan S. J., Henning T., Schreyer K., 1996, A\&AS, 115, 285

Chavarría L. A., Allen L. E., Hora J. L., Brunt C. M., Fazio

G. G., 2008, ApJ, 682, 445

Churchwell E. et al., 2006, ApJ, 649, 759

Churchwell E. et al., 2007, ApJ, 670, 428

Comerón F., Pasquali A., 2012, A\&A, 543, A101

Contreras Y., Garay G., Rathborne J. M., Sanhueza P., 2016, MNRAS, 456, 2041

Curiel S., Rodriguez L. F., Moran J. M., Canto J., 1993, ApJ, 415, 191

Deharveng L. et al., 2010, A\&A, 523, A6

Deller A. T., Forbrich J., Loinard L., 2013, A\&A, 552, A51

Dewangan L. K., Ojha D. K., 2013, MNRAS, 429, 1386

Dewangan L. K., Ojha D. K., Anandarao B. G., Ghosh

S. K., Chakraborti S., 2012, ApJ, 756, 151

Dyson J. E., Williams D. A., 1980, Physics of the interstellar medium

Elmegreen B. G., Lada C. J., 1977, ApJ, 214, 725

Faimali A. et al., 2012, MNRAS, 426, 402

Foster J. B. et al., 2011, ApJS, 197, 25

Foster J. B. et al., 2013, PASA, 30, e038

Garay G., Moran J. M., Rodríguez L. F., Reid M. J., 1998, ApJ, 492, 635

Garwood R. W., Dickey J. M., 1989, ApJ, 338, 841

Goodman A. A., Rosolowsky E. W., Borkin M. A., Foster J. B., Halle M., Kauffmann J., Pineda J. E., 2009, Nature, 457, 63

Griffin M. J. et al., 2010, A\&A, 518, L3

Guieu S. et al., 2010, ApJ, 720, 46

Gutermuth R. A., Heyer M., 2015, AJ, 149, 64

Haslam C. G. T., Salter C. J., Stoffel H., Wilson W. E., 1982, A\&AS, 47, 1

Hattori Y. et al., 2016, PASJ, 68, 37

Hildebrand R. H., 1983, QJRAS, 24, 267

Hou L. G., Gao X. Y., 2014, MNRAS, 438, 426

Inoue A. K., 2001, AJ, 122, 1788

Jackson J. M. et al., 2013, PASA, 30, e057

Ji W.-G., Zhou J.-J., Esimbek J., Wu Y.-F., Wu G., Tang X.-D., 2012, A\&A, 544, A39

Kauffmann J., Bertoldi F., Bourke T. L., Evans, II N. J., Lee C. W., 2008, A\&A, 487, 993

Kauffmann J., Pillai T., 2010, ApJ, 723, L7

Kendrew S. et al., 2012, ApJ, 755, 71

Kim K.-T., Koo B.-C., 2001, ApJ, 549, 979

Kim K.-T., Koo B.-C., 2003, ApJ, 596, 362

Kolpak M. A., Jackson J. M., Bania T. M., Clemens D. P., Dickey J. M., 2003, ApJ, 582, 756

Kwan J., 1997, ApJ, 489, 284

Lada C. J., 1987, in IAU Symposium, Vol. 115, Star Forming Regions, Peimbert M., Jugaku J., eds., pp. 1-17

Lada C. J., Adams F. C., 1992, ApJ, 393, 278
Ladd N., Purcell C., Wong T., Robertson S., 2005, PASA, 22,62

Lal D. V., Rao A. P., 2007, MNRAS, 374, 1085

Launhardt R. et al., 2013, A\&A, 551, A98

Lefloch B., Lazareff B., 1994, A\&A, 289, 559

Liu H.-L. et al., 2017a, A\&A, 602, A95

Liu H.-L. et al., 2016, ApJ, 818, 95

Liu H.-L., Wu Y., Li J., Yuan J.-H., Liu T., Dong X., 2015, ApJ, 798, 30

Liu T. et al., 2017b, ArXiv e-prints

Liu T., Wu Y., Zhang H., Qin S.-L., 2012, ApJ, 751, 68

Luque-Escamilla P. L., Muñoz-Arjonilla A. J., SánchezSutil J. R., Martí J., Combi J. A., Sánchez-Ayaso E., 2011, A\&A, 532, A92

Mallick K. K., Ojha D. K., Tamura M., Linz H., Samal M. R., Ghosh S. K., 2015, MNRAS, 447, 2307

Martín-Hernández N. L., van der Hulst J. M., Tielens A. G. G. M., 2003, A\&A, 407, 957

Martins F., Plez B., 2006, A\&A, 457, 637

Martins F., Schaerer D., Hillier D. J., 2005, A\&A, 436, 1049

Marton G. et al., 2017, ArXiv e-prints

Meyer M. R., Calvet N., Hillenbrand L. A., 1997, AJ, 114, 288

Miettinen O., 2014, A\&A, 562, A3

Molinari S. et al., 2010, A\&A, 518, L100

Müller H. S. P., Schlöder F., Stutzki J., Winnewisser G., 2005, Journal of Molecular Structure, 742, 215

Müller H. S. P., Thorwirth S., Roth D. A., Winnewisser G., 2001, A\&A, 370, L49

Nandakumar G., Veena V. S., Vig S., Tej A., Ghosh S. K., Ojha D. K., 2016, AJ, 152, 146

Ochsendorf B. B., Verdolini S., Cox N. L. J., Berné O., Kaper L., Tielens A. G. G. M., 2014, A\&A, 566, A75

Ojha D. K. et al., 2004a, ApJ, 608, 797

Ojha D. K. et al., 2004b, ApJ, 616, 1042

Ortega M. E., Giacani E., Paron S., Rubio M., 2016, MNRAS, 458, 3684

Panagia N., 1973, AJ, 78, 929

Paron S., Petriella A., Ortega M. E., 2011, A\&A, 525, A132

Peeters E., Hony S., Van Kerckhoven C., Tielens A. G. G. M., Allamandola L. J., Hudgins D. M., Bauschlicher C. W., 2002, A\&A, 390, 1089

Peretto N. et al., 2010, A\&A, 518, L98

Petriella A., Paron S., Giacani E., 2010, A\&A, 513, A44

Poglitsch A. et al., 2010, A\&A, 518, L2

Pomarès M. et al., 2009, A\&A, 494, 987

Povich M. S. et al., 2007, ApJ, 660, 346

Purcell C. R. et al., 2006, MNRAS, 367, 553

Quireza C., Rood R. T., Bania T. M., Balser D. S., Maciel

W. J., 2006, ApJ, 653, 1226

Ranjan Das S., Tej A., Vig S., Ghosh S. K., Ishwara Chandra C. H., 2016, AJ, 152, 152

Rathborne J. M. et al., 2014, ApJ, 786, 140

Rebull L. M. et al., 2011, ApJS, 196, 4

Rieke G. H., Lebofsky M. J., 1985, ApJ, 288, 618

Rieke G. H. et al., 2004, ApJS, 154, 25

Rodriguez L. F., Curiel S., Moran J. M., Mirabel I. F., Roth M., Garay G., 1989, ApJ, 346, L85

Rodríguez L. F., Masqué J. M., Dzib S. A., Loinard L., Kurtz S. E., 2014, Rev. Mexicana Astron. Astrofis., 50, 3 Roger R. S., Costain C. H., Landecker T. L., Swerdlyk C. M., 1999, A\&AS, 137, 7 
Roman-Duval J., Jackson J. M., Heyer M., Johnson A., Rathborne J., Shah R., Simon R., 2009, ApJ, 699, 1153

Rosero V. et al., 2016, ApJS, 227, 25

Rosolowsky E. W., Pineda J. E., Kauffmann J., Goodman A. A., 2008, ApJ, 679, 1338

Russeil D. et al., 2013, A\&A, 554, A42

Russeil D. et al., 2016, A\&A, 587, A135

Sánchez-Monge Á., Beltrán M. T., Cesaroni R., Fontani F., Brand J., Molinari S., Testi L., Burton M., 2013a, A\&A, 550, A21

Sánchez-Monge Á., Kurtz S., Palau A., Estalella R., Shepherd D., Lizano S., Franco J., Garay G., 2013b, ApJ, 766, 114

Sanhueza P., Jackson J. M., Foster J. B., Garay G., Silva A., Finn S. C., 2012, ApJ, 756, 60

Schmiedeke A. et al., 2016, A\&A, 588, A143

Schneider N. et al., 2013, ApJ, 766, L17

Schneider N. et al., 2012, A\&A, 540, L11

Schneider N. et al., 2015, A\&A, 575, A79

Schöier F. L., van der Tak F. F. S., van Dishoeck E. F., Black J. H., 2005, A\&A, 432, 369

Sidorin V., Douglas K. A., Palouš J., Wünsch R., Ehlerová S., 2014, A\&A, 565, A6

Simpson R. J. et al., 2012, MNRAS, 424, 2442

Skrutskie M. F. et al., 2006, AJ, 131, 1163

Spitzer L., 1978, Physical processes in the interstellar medium

Sugitani K. et al., 2002, ApJ, 565, L25

Swarup G., Ananthakrishnan S., Kapahi V. K., Rao A. P., Subrahmanya C. R., Kulkarni V. K., 1991, Current Science, Vol. 60, NO.2/JAN25, P. 95, 1991, 60, 95

Tan J. C., Beltrán M. T., Caselli P., Fontani F., Fuente A., Krumholz M. R., McKee C. F., Stolte A., 2014, Protostars and Planets VI, 149

Tej A., Ojha D. K., Ghosh S. K., Kulkarni V. K., Verma R. P., Vig S., Prabhu T. P., 2006, A\&A, 452, 203

Thompson M. A., Urquhart J. S., Moore T. J. T., Morgan L. K., 2012, MNRAS, 421, 408

Tremblin P., Audit E., Minier V., Schmidt W., Schneider N., 2012a, A\&A, 546, A33

Tremblin P., Audit E., Minier V., Schneider N., 2012b, A\&A, 538, A31

Tremblin P. et al., 2014, A\&A, 564, A106

Urquhart J. S. et al., 2013, MNRAS, 431, 1752

Urquhart J. S., Thompson M. A., Morgan L. K., Pestalozzi M. R., White G. J., Muna D. N., 2007, A\&A, 467, 1125

van der Tak F. F. S., Black J. H., Schöier F. L., Jansen D. J., van Dishoeck E. F., 2007, A\&A, 468, 627

Vasyunina T., Linz H., Henning T., Zinchenko I., Beuther H., Voronkov M., 2011, A\&A, 527, A88

Veena V. S., Vig S., Tej A., Varricatt W. P., Ghosh S. K., Chandrasekhar T., Ashok N. M., 2016, MNRAS, 456, 2425

Vig S., Ghosh S. K., Ojha D. K., Verma R. P., 2007, A\&A, 463, 175

Watson C., Corn T., Churchwell E. B., Babler B. L., Povich M. S., Meade M. R., Whitney B. A., 2009, ApJ, 694, 546 Watson C. et al., 2008, ApJ, 681, 1341

Weaver R., McCray R., Castor J., Shapiro P., Moore R., 1977, ApJ, 218, 377

Whitworth A. P., Bhattal A. S., Chapman S. J., Disney M. J., Turner J. A., 1994, MNRAS, 268, 291

Williams J. P., de Geus E. J., Blitz L., 1994, ApJ, 428, 693
Yu N., Wang J.-J., 2015, MNRAS, 451, 2507

Yu N., Xu J., 2016, ApJ, 833, 248

Zacharias N., Monet D. G., Levine S. E., Urban S. E., Gaume R., Wycoff G. L., 2004, in Bulletin of the American Astronomical Society, Vol. 36, American Astronomical Society Meeting Abstracts, p. 1418

Zavagno A. et al., 2010, A\&A, 518, L101

Zinnecker H., Yorke H. W., 2007, ARA\&A, 45, 481

Zoonematkermani S., Helfand D. J., Becker R. H., White R. L., Perley R. A., 1990, ApJS, 74, 181

\section{APPENDIX A: YSO LIST.}


Table A1. List of identified YSOs detected in region of bubble CS51. The 2MASS and IRAC photometric magnitudes of the YSOs are given.

\begin{tabular}{|c|c|c|c|c|}
\hline YSO & $\begin{array}{c}\mathrm{RA} \\
(h\end{array}$ & 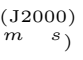 & $\begin{array}{c}\text { DEC } \\
\left({ }^{\circ}\right.\end{array}$ & (J \\
\hline 1 & 173 & 051.95 & -33 & 52 \\
\hline 2 & 173 & 053.83 & -33 & 54 \\
\hline 3 & 173 & 054.05 & -33 & 51 \\
\hline 4 & 173 & 54.77 & -33 & 50 \\
\hline 5 & 173 & 055.05 & -33 & 50 \\
\hline 6 & 173 & 56.78 & -33 & 54 \\
\hline 7 & 173 & 58.02 & -33 & 54 \\
\hline 8 & 173 & 58.15 & -33 & 503 \\
\hline 9 & 173 & 58.38 & -33 & 55 \\
\hline 10 & 173 & 059.46 & -33 & 52 \\
\hline 11 & 173 & 059.52 & -33 & 54 \\
\hline 12 & 173 & $\begin{array}{lll}1 & 01.19\end{array}$ & -33 & 51 \\
\hline 13 & 173 & $\begin{array}{lll}1 & 01.35\end{array}$ & -33 & 50 \\
\hline 14 & 173 & $\begin{array}{lll}1 & 01.35\end{array}$ & -33 & 51 \\
\hline 15 & 173 & 101.97 & -33 & 54 \\
\hline 16 & 173 & $\begin{array}{ll}1 & 02.12\end{array}$ & -33 & 53 \\
\hline 17 & 173 & $\begin{array}{ll}1 & 03.24\end{array}$ & -33 & 53 \\
\hline 18 & 173 & $\begin{array}{lll}1 & 03.37\end{array}$ & -33 & $53=$ \\
\hline 19 & 173 & 103.79 & -33 & 51 \\
\hline 20 & 173 & $\begin{array}{lll}1 & 03.87\end{array}$ & -33 & 49 \\
\hline 21 & 173 & $\begin{array}{lll}1 & 03.91\end{array}$ & -33 & 50 \\
\hline 22 & 173 & $\begin{array}{lll}1 & 03.94\end{array}$ & -33 & 50 \\
\hline 23 & 173 & $\begin{array}{lll}1 & 03.96\end{array}$ & -33 & 483 \\
\hline 24 & 173 & 104.63 & -33 & 54 \\
\hline 25 & 173 & $\begin{array}{lll}1 & 05.47\end{array}$ & -33 & 53 \\
\hline 26 & 173 & 106.01 & -33 & 50 \\
\hline 27 & 173 & 106.37 & -33 & 51 \\
\hline 28 & 173 & $\begin{array}{lll}1 & 06.46\end{array}$ & -33 & 55 \\
\hline 29 & 173 & 107.16 & -33 & 50 \\
\hline 30 & 173 & $\begin{array}{lll}1 & 07.17\end{array}$ & -33 & 52 \\
\hline 31 & 173 & $\begin{array}{lll}1 & 07.57\end{array}$ & -33 & 52 \\
\hline 32 & 173 & $\begin{array}{lll}1 & 07.80\end{array}$ & -33 & 54 \\
\hline 33 & 173 & $\begin{array}{lll}1 & 07.94\end{array}$ & -33 & 49 \\
\hline 34 & 173 & 108.08 & -33 & 50 \\
\hline 35 & 173 & $\begin{array}{ll}1 & 08.22\end{array}$ & -33 & 48 \\
\hline 36 & 173 & $\begin{array}{lll}1 & 08.91\end{array}$ & -33 & 48 \\
\hline 37 & 173 & $\begin{array}{ll}1 & 09.17\end{array}$ & -33 & 54 \\
\hline 38 & 173 & $\begin{array}{lll}1 & 09.33\end{array}$ & -33 & $48=$ \\
\hline 39 & 173 & 109.34 & -33 & 56 \\
\hline 40 & 173 & 109.61 & -33 & 54 \\
\hline 41 & 173 & $\begin{array}{lll}1 & 09.67\end{array}$ & -33 & 55 \\
\hline 42 & 173 & $\begin{array}{ll}1 & 09.87\end{array}$ & -33 & 53 \\
\hline 43 & 173 & 110.38 & -33 & 56 \\
\hline 44 & 173 & $\begin{array}{ll}1 & 10.59\end{array}$ & -33 & 49 \\
\hline 45 & 173 & 110.67 & -33 & 56 \\
\hline 46 & 173 & 112.25 & -33 & 483 \\
\hline 47 & 173 & $1 \quad 12.44$ & -33 & 50 \\
\hline 48 & 173 & 12.84 & -33 & 49 \\
\hline $\begin{array}{l}48 \\
49\end{array}$ & 173 & $\begin{array}{ll}1 & 12.84 \\
1 & 13.04\end{array}$ & -33 & 50 \\
\hline 50 & 173 & 114.78 & -33 & 515 \\
\hline 51 & 173 & 114.84 & -33 & 48 \\
\hline 52 & 173 & 15.43 & -33 & 56 \\
\hline 53 & 173 & $\begin{array}{ll}1 & 15.75\end{array}$ & -33 & 50 \\
\hline 54 & 173 & 16.18 & -33 & 54 \\
\hline 55 & 173 & 116.38 & -33 & $53 \mathrm{c}$ \\
\hline 56 & 173 & 17.28 & -33 & 513 \\
\hline 57 & 173 & 17.48 & -33 & 52 \\
\hline 58 & 173 & 18.85 & -33 & 55 \\
\hline 59 & 173 & $\begin{array}{ll}1 & 18.00\end{array}$ & -33 & 57 \\
\hline 60 & 173 & 119.45 & -33 & 52 \\
\hline 61 & 173 & 19.58 & -33 & 51 \\
\hline 62 & 173 & 119.66 & -33 & 543 \\
\hline 63 & 173 & $\begin{array}{ll}1 & 20.14\end{array}$ & -33 & 48 \\
\hline 64 & 173 & 120.26 & -33 & 57 \\
\hline 65 & 173 & 120.39 & -33 & 54 \\
\hline 66 & 173 & 121.26 & -33 & 503 \\
\hline 67 & 173 & $\begin{array}{ll}1 & 21.38\end{array}$ & -33 & 57 \\
\hline 68 & 173 & 121.57 & -33 & 51 \\
\hline 69 & 173 & 122.86 & -33 & 55 \\
\hline 70 & 173 & $\begin{array}{lll}1 & 23.48\end{array}$ & -33 & 54 \\
\hline 71 & 173 & 124.40 & -33 & 562 \\
\hline 72 & 173 & $\begin{array}{lll}1 & 24.41\end{array}$ & -33 & 55 \\
\hline 73 & 173 & 125.33 & -33 & 49 \\
\hline 74 & 173 & 126.66 & -33 & 52 \\
\hline 75 & 173 & 126.86 & -33 & 54 \\
\hline 76 & 173 & 127.04 & -33 & 54 \\
\hline 77 & 173 & 127.43 & -33 & 53 \\
\hline 78 & 173 & 127.87 & -33 & 503 \\
\hline 79 & 173 & 128.41 & -33 & 513 \\
\hline 80 & 173 & 128.43 & -33 & 54 \\
\hline & & 128.57 & & 503 \\
\hline 82 & 173 & 128.80 & -33 & 56 \\
\hline 83 & 173 & 128.98 & -33 & 512 \\
\hline 84 & 173 & 130.39 & -33 & 543 \\
\hline 85 & 173 & 131.03 & -33 & 50 \\
\hline $\begin{array}{l}80 \\
86\end{array}$ & 173 & $\begin{array}{lll}1 & 31.00 \\
1 & 31.15\end{array}$ & -33 & 54 \\
\hline 87 & 173 & 131.27 & -33 & 55 \\
\hline 88 & & $\begin{array}{lll}1 & 32.79\end{array}$ & & 54 \\
\hline 89 & 173 & 133.47 & -33 & 54 \\
\hline 90 & 173 & 134.96 & -33 & 52 \\
\hline & & 135.17 & & 50 \\
\hline 92 & 173 & $\begin{array}{lll}1 & 35.71\end{array}$ & -33 & 525 \\
\hline 93 & 173 & 137.18 & -33 & 523 \\
\hline
\end{tabular}

